\title{
Identification and developmental expression of the full complement of Cytochrome P450 genes in Zebrafish
}

Jared V Goldstone ${ }^{1}$, Andrew G McArthur ${ }^{2}$, Akira Kubota ${ }^{1}$, Juliano Zanette ${ }^{1,3}$, Thiago Parente ${ }^{1,4}$, Maria E Jönsson ${ }^{1,5}$, David R Nelson ${ }^{6}$, John J Stegeman ${ }^{1 *}$

\begin{abstract}
Background: Increasing use of zebrafish in drug discovery and mechanistic toxicology demands knowledge of cytochrome P450 (CYP) gene regulation and function. CYP enzymes catalyze oxidative transformation leading to activation or inactivation of many endogenous and exogenous chemicals, with consequences for normal physiology and disease processes. Many CYPs potentially have roles in developmental specification, and many chemicals that cause developmental abnormalities are substrates for CYPs. Here we identify and annotate the full suite of CYP genes in zebrafish, compare these to the human CYP gene complement, and determine the expression of CYP genes during normal development.
\end{abstract}

Results: Zebrafish have a total of 94 CYP genes, distributed among 18 gene families found also in mammals. There are 32 genes in CYP families 5 to 51, most of which are direct orthologs of human CYPs that are involved in endogenous functions including synthesis or inactivation of regulatory molecules. The high degree of sequence similarity suggests conservation of enzyme activities for these CYPs, confirmed in reports for some steroidogenic enzymes (e.g. CYP19, aromatase; CYP11A, P450scc; CYP17, steroid 17a-hydroxylase), and the CYP26 retinoic acid hydroxylases. Complexity is much greater in gene families 1, 2, and 3, which include CYPs prominent in metabolism of drugs and pollutants, as well as of endogenous substrates. There are orthologous relationships for some CYP1 s and some CYP3 s between zebrafish and human. In contrast, zebrafish have 47 CYP2 genes, compared to 16 in human, with only two (CYP2R1 and CYP2U1) recognized as orthologous based on sequence. Analysis of shared synteny identified CYP2 gene clusters evolutionarily related to mammalian CYP2 s, as well as unique clusters.

Conclusions: Transcript profiling by microarray and quantitative PCR revealed that the majority of zebrafish CYP genes are expressed in embryos, with waves of expression of different sets of genes over the course of development. Transcripts of some CYP occur also in oocytes. The results provide a foundation for the use of zebrafish as a model in toxicological, pharmacological and chemical disease research.

\section{Background}

The cytochrome P450 (CYP) enzymes catalyze oxidative transformation leading to activation or inactivation of many endogenous and exogenous chemicals, with consequences for normal physiology and disease processes. Mammalian CYPs can be separated into two major groups: those with generally narrow substrate specificity

\footnotetext{
* Correspondence: jstegeman@whoi.edu

'Biology Department, Woods Hole Oceanographic Institution, Woods Hole, MA, USA

Full list of author information is available at the end of the article
}

that are involved primarily in synthesis, activation or inactivation of endogenous regulatory molecules, and those involved most heavily in the metabolism of xenobiotics, but which may act as well in the metabolism of endogenous compounds. Thus, CYP enzymes can determine the persistence and action of endogenous regulatory molecules as well as many drugs and other toxicants and carcinogens.

An area growing in importance is the role of CYPs in development, and in developmental toxicity of chemicals. Absolute embryo lethality upon knockout of the

\section{() Biomed Central}


murine CYP oxidoreductase (Por) demonstrates the essential role of CYP enzymes in murine development [1]. Many CYPs potentially have roles in developmental specification. For example, it is well known that CYP26 enzymes regulate levels of retinoids governing pattern formation during development [2-4]. CYP isoforms that play essential roles in bile acid homeostasis, steroidogenesis, the vitamin D pathway, and the catabolism of many hormones may also have important roles in development [5-12].

Many chemicals that cause developmental abnormalities, including cardiovascular, neural, and connective tissue defects, are substrates for CYPs, and the oxidative biotransformation of such xenobiotics may determine the cellular and organ targets of those chemicals [12]. CYP enzymes that are prominent in xenobiotic metabolism could also function in producing morphogenic molecules or keeping regions free of them, creating temporal and spatial regions of morphogen action and supporting region-specific changes essential for successful development [12-14]. The roles and regulation of most xenobiotic metabolizing CYPs during development are unknown, impeding understanding of mechanisms of developmental toxicity. Many xenobiotic metabolizing CYP can be induced via transcription factors (aryl hydrocarbon receptor, AHR; pregnane-X-receptor, PXR, peroxisome proliferator activated receptors, PPARs; and others $[15,16])$. All CYPs can be targets of exogenous chemicals that disrupt or enhance their function, whether or not they are directly involved in xenobiotic metabolism.

The zebrafish has emerged as one of the most important vertebrate model species in embryology and developmental biology $[17,18]$, due largely to rapid development coupled with the possibility for genetic analysis and manipulation. Multiple draft assemblies of the zebrafish genome have been produced by the Wellcome Trust Sanger Institute [19]. The near completion of the zebrafish genome has made it possible to address questions of CYP gene identity and expression during zebrafish development with substantial clarity and completeness.

Our objective was to identify and annotate the full suite of zebrafish CYP genes and examine their phylogenetic and shared syntenic relationships to human CYPs. We also examined CYP expression during early development in zebrafish. CYP expression in vertebrate development has not been completely mapped for any species; prior studies in mice examined developmental expression of 40 CYP genes $[10,20]$, less than half of the mouse CYP complement [21]. Our analyses identify novel genes and clusters of CYP genes in zebrafish, and unanticipated waves of CYP expression during development.

\section{Results}

Repeated and exhaustive searching of zebrafish genome assemblies uncovered a total of 94 CYP genes. Based on inferred amino acid sequences, these genes fell into 18 CYP gene families that are also found as well in humans and other mammals. Molecular phylogenetic analysis (Figure 1) shows relationships among CYP genes and gene families in zebrafish and human. As with the human CYP genes, the CYP genes in zebrafish occur in two major functional groups: those families that include enzymes involved primarily in endogenous functions (CYP families 5-51; Table 1); and those that include enzymes involved heavily in oxidation of xenobiotics (CYP families 1-3, and to a lesser degree family 4; Table 2). Zebrafish genes in these two major groups are described in detail below, considering relationship to human CYPs based on sequence identity, syntenic analysis and, where known, functional properties (functions of human or other mammalian homologs [22] are indicated in parentheses). The term CYP is fully capitalized in all cases except for mouse, rat, and Drosophila (where only the first letter is capitalized), and italicized only when referring to a specific gene or cDNA, consistent with the nomenclature committee recommendations [23].

\section{CYP Families 5-51}

\section{CYP5 (thromboxane A2 synthase, TBXAS1}

The mammalian CYP5A1 enzyme catalyzes rearrangement of prostaglandin $\mathrm{H} 2$ (PGH2). Zebrafish CYP5A1 retains $48 \%$ sequence identity with human $C Y P 5 A 1$, and is located in a region of shared synteny with $C Y P 5 A 1$ in humans, indicating orthology.

\section{CYP7 (steroid 7 $\alpha$-hydroxylase)}

Zebrafish possess one six-exon $C Y P 7 A 1$ and one sixexon $C Y P 7 B 1$ gene, both located on Chromosome 2. The sequence identity between zebrafish $C Y P 7 B 1$ and mammalian $C Y P 7 B s$ technically precludes membership in the same subfamily, yet shared synteny (Table 3) indicates orthology. Zebrafish also have a third CYP7 gene, $C Y P 7 C 1$, a $C Y P 7 A$-like gene located on Chromosome 7, possibly an ohnolog resulting from the fish-specific whole genome duplication (WGD 3; [24]).

\section{CYP8 (prostacyclin synthase, PTGIS)}

A 10-exon zebrafish CYP8A1 gene is located on chromosome 6. Human CYP8A1 catalyzes the rearrangement of PGH2 to prostaglandin I2 (prostacyclin) [25]. A crystal structure of zebrafish CYP8A1 published in 2008 as part of a comparative structural analysis of prostacyclin synthases, shows that zebrafish and human CYP8A1 exhibit nearly identical ligand-bound and ligand-free three-dimensional structures, despite exhibiting only $45 \%$ identity [26]. Zebrafish also possess three CYP8Bs 


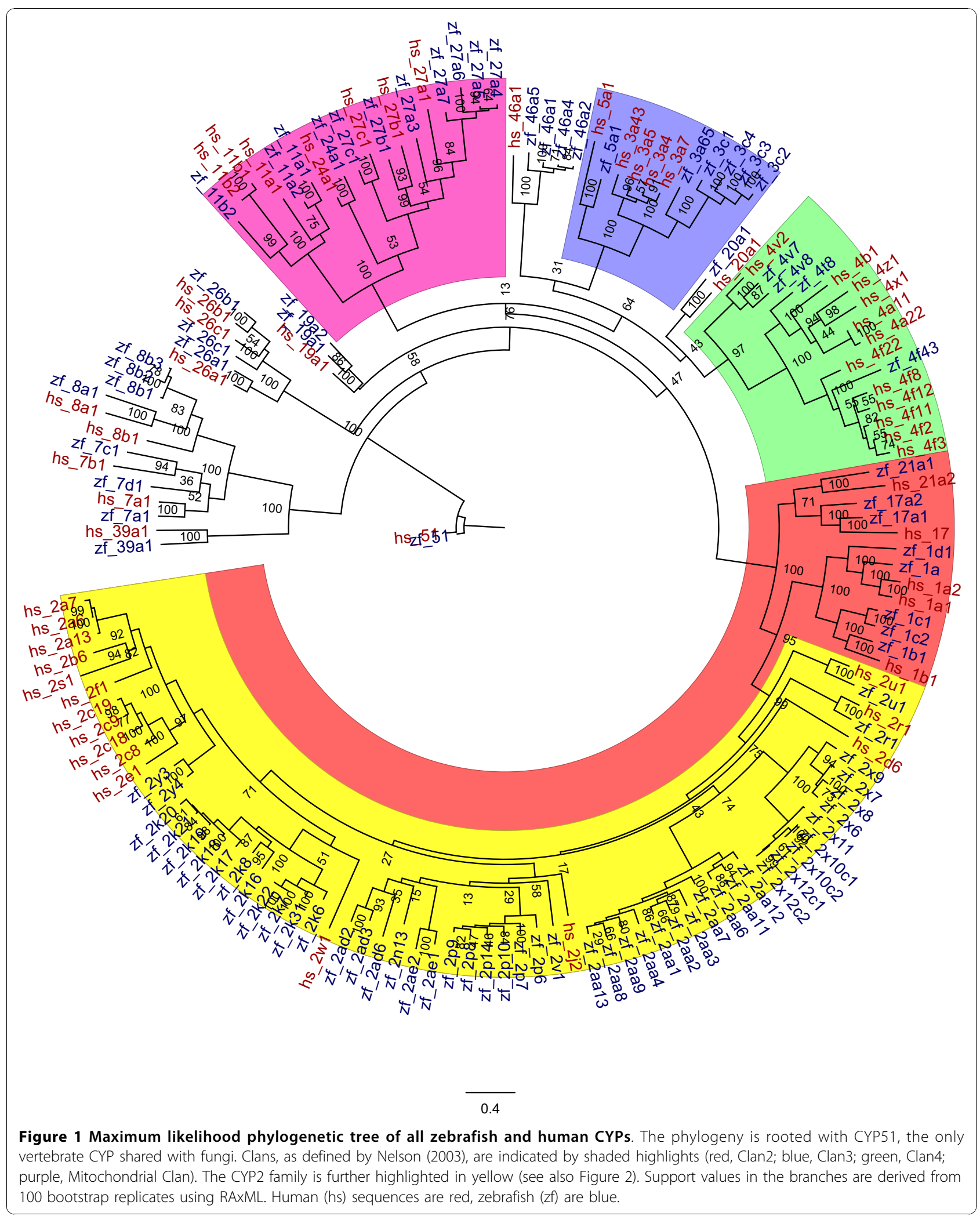


Table 1 Zebrafish CYPs in families 5-51, with Chromosomal location (Zv8), Ensembl gene ID (Release 58), and exon count

\begin{tabular}{|c|c|c|c|c|c|c|c|c|c|}
\hline $\begin{array}{l}\text { Gene } \\
\text { name }\end{array}$ & $\begin{array}{l}\text { Ensembl } 58 \\
\text { Gene ID }\end{array}$ & $\begin{array}{l}\text { Ensembl } 58 \\
\text { Transcript ID }\end{array}$ & $\begin{array}{l}\text { Ensembl } 58 \\
\text { Protein ID }\end{array}$ & Chromosome & $\begin{array}{l}\text { Transcript } \\
\text { Start (bp) }\end{array}$ & $\begin{array}{r}\text { Transcript } \\
\text { End (bp) }\end{array}$ & Strand & Exon & Ref \\
\hline CYP5A1 & ENSDARG00000002249 & ENSDART00000109497 & ENSDARP00000100741 & 18 & 12577084 & 12755668 & 1 & 13 & \\
\hline CYP7A1 & ENSDARG00000069018 & ENSDART00000100069 & ENSDARP00000090841 & 2 & 20390259 & 20392545 & -1 & 6 & \\
\hline CYP7C1 & ENSDARG00000008858 & ENSDART00000008268 & ENSDARP00000020000 & 2 & 41226478 & 41238852 & 1 & 5 & \\
\hline CYP7D1 & ENSDARG00000004051 & ENSDART00000012822 & ENSDARP00000007208 & 7 & 60786672 & 60803272 & -1 & 6 & \\
\hline CYP8A1 & ENSDARG00000060094 & ENSDART00000084334 & ENSDARP00000078769 & 6 & 50097042 & 50118485 & -1 & 10 & [26] \\
\hline CYP8B1 & ENSDARG00000053068 & ENSDART00000053012 & ENSDARP00000091773 & 2 & 2631931 & 2633902 & -1 & 1 & \\
\hline CYP8B2 & ENSDARG00000059008 & ENSDART00000053010 & listed as pseudogene & 2 & 2598906 & 26000260 & -1 & 1 & \\
\hline CYP8B3 & ENSDARG00000059000 & ENSDART00000012143 & listed as pseudogene & 2 & 2583060 & 2584972 & -1 & 1 & \\
\hline CYP11A1 & ENSDARG00000002347 & ENSDART00000024866 & ENSDARP00000025030 & 25 & 23348335 & 23363675 & 1 & 11 & {$[117]$} \\
\hline CYP11A2 & ENSDARG00000051860 & ENSDART00000073566 & ENSDARP00000068056 & 25 & 23325076 & 23339692 & 1 & 9 & \\
\hline CYP11C1 & ENSDARG00000042014 & ENSDART00000061572 & ENSDARP00000061571 & 16 & 51516891 & 51525686 & 1 & 10 & \\
\hline CYP17A1 & ENSDARG00000033566 & ENSDART00000043156 & ENSDARP00000043155 & 13 & 33337340 & 33347115 & 1 & 8 & {$[29]$} \\
\hline CYP17A2 & ENSDARG00000053966 & ENSDART00000076086 & ENSDARP00000070565 & 23 & 44130651 & 44138933 & 1 & 9 & {$[32]$} \\
\hline CYP19A1 & ENSDARG00000041348 & ENSDART00000060605 & ENSDARP00000060604 & 18 & 39131244 & 39146992 & -1 & 9 & {$[34]$} \\
\hline CYP19A2 & ENSDARG00000009852 & ENSDART00000025590 & ENSDARP00000014320 & 25 & 3756544 & 3767668 & 1 & 10 & {$[34]$} \\
\hline CYP20A1 & ENSDARG00000006040 & ENSDART00000019325 & ENSDARP00000003222 & 5 & 61726392 & 61743655 & -1 & 15 & \\
\hline CYP21A1 & ENSDARG00000037550 & ENSDART00000059833 & ENSDARP00000059832 & 16 & 15091143 & 15104788 & -1 & 12 & \\
\hline CYP24A1 & ENSDARG00000070420 & ENSDART00000103307 & ENSDARP00000094084 & Zv8_NA3215 & 47572 & 49579 & -1 & 12 & \\
\hline CYP26A1 & ENSDARG00000033999 & ENSDART00000041728 & ENSDARP00000041727 & 12 & 9329895 & 9333488 & -1 & 7 & {$[41]$} \\
\hline CYP26B1 & ENSDARG00000077121 & ENSDART00000110347 & ENSDARP00000101543 & 7 & 25393384 & 25417689 & 1 & 6 & {$[118]$} \\
\hline CYP26C1 ${ }^{d}$ & ENSDARG00000056029 & ENSDART00000077809 & ENSDARP00000072275 & 17 & 17123846 & 17136305 & 1 & 7 & {$[$ [119] } \\
\hline CYP27A3 & ENSDARG00000057262 & ENSDART00000079879 & ENSDARP00000074330 & 9 & 22363398 & 22377078 & 1 & 10 & \\
\hline CYP27A4 & ENSDARG00000055159 & ENSDART00000077479 & ENSDARP00000071946 & 9 & 39232071 & 39242971 & 1 & 9 & \\
\hline CYP27A5 & ENSDARG00000055159 & ENSDART00000108860 & ENSDARP00000101333 & 9 & 39213139 & 39219475 & 1 & 9 & \\
\hline CYP27A6 & ENSDARG00000069186 & ENSDART00000100487 & ENSDARP00000091260 & 9 & 39205591 & 39211049 & 1 & 9 & \\
\hline CYP27A7 & ENSDARG00000033802 & ENSDART00000045588 & ENSDARP00000045587 & 9 & 39194558 & 39204706 & 1 & 9 & \\
\hline CYP27B1 & ENSDARG00000045015 & ENSDART00000066178 & ENSDARP00000066177 & 11 & 460256 & 466291 & -1 & 11 & \\
\hline CYP27C1 & ENSDARG00000058439 & ENSDART00000081309 & ENSDARP00000075752 & 6 & 10507141 & 10520886 & -1 & 9 & \\
\hline CYP39A1 & ENSDARG00000017982 & ENSDART00000005764 & ENSDARP00000016128 & 20 & 39084490 & 39112294 & -1 & 12 & \\
\hline CYP46A1 & ENSDARG00000012137 & ENSDART00000028039 & ENSDARP00000025234 & 20 & 4947206 & 4961574 & 1 & 15 & \\
\hline CYP46A2 & ENSDARG00000004262 & ENSDART00000057699 & ENSDARP00000057698 & 5 & 73217357 & 73226060 & -1 & 15 & \\
\hline CYP46A4 & ENSDARG00000039492 & ENSDART00000099258 & ENSDARP00000090030 & 5 & 73235710 & 73241459 & -1 & 15 & \\
\hline CYP46A5 & not in & Ensembl & Build $58^{e}$ & & & & & & \\
\hline CYP51A1 & ENSDARG00000042641 & ENSDART00000062551 & ENSDARP00000062550 & 19 & 486980 & 505501 & 1 & 10 & {$[54]$} \\
\hline
\end{tabular}

(CYP8B1-CYP8B3), which are single-exon genes located on Chromosome 2 that share synteny with the singleexon human CYP8B1.

\section{CYP11 (pregnenolone and aldosterone synthases)}

Zebrafish have 2 CYP11A genes (side-chain cleavage enzymes), CYP11A1 and CYP11A2, located adjacent to one another on chromosome 25, and one CYP11B-like gene, termed CYP11C1 (on Chromosome 16). Vertebrate CYP11A1 synthesizes pregnenolone from cholesterol, and a maternally-derived CYP11A1 mRNA has been suggested to form pregnenolone important in cell migration in the zebrafish zygote [27]. Following the onset of embryonic transcription, $C Y P 11 A 1$ is expressed in the yolk syncytial layer. It is then expressed in the embryonic interrenal primordia, and in the interrenal glands into adulthood $[5,27,28]$. In adult zebrafish it is expressed in the gonads, brain, and interrenal glands. Knockdown of zebrafish CYP11A1 leads to a shortened axis and epiboly 
Table 2 Zebrafish CYPs in families 1-4, with Chromosomal location (Zv8), Ensembl gene ID (Release 58), and exon count

\begin{tabular}{|c|c|c|c|c|c|c|c|c|c|}
\hline Gene name & $\begin{array}{l}\text { Ensembl } 58 \\
\text { Gene ID }\end{array}$ & $\begin{array}{l}\text { Ensembl } 58 \\
\text { Transcript ID }\end{array}$ & $\begin{array}{l}\text { Ensembl } 58 \\
\text { Protein ID }\end{array}$ & Chromosome & $\begin{array}{l}\text { Transcript } \\
\text { Start (bp) }\end{array}$ & $\begin{array}{l}\text { Transcript } \\
\text { End (bp) }\end{array}$ & Strand & Exon & Ref \\
\hline CYP1A & ENSDARG000000026039 & ENSDART000000038200 & ENSDARP000000033498 & 18 & 3972990 & 3983879 & -1 & 7 & {$[56]$} \\
\hline CYP1B1 & ENSDARG000000068934 & ENSDART00000099870 & ENSDARP000000090643 & 13 & 42451108 & 42458759 & -1 & 2 & [62] \\
\hline CYP1C1 & ENSDARG000000058980 & ENSDART000000019953 & ENSDARP000000004126 & Zv8_scaf 3050 & 158017 & 161082 & 1 & 1 & {$[62]$} \\
\hline CYP1C2 & ENSDARG00000018298 & ENSDART000000016487 & ENSDARP00000019538 & Zv8_scaf 3050 & 152452 & 154638 & 1 & 1 & {$[62]$} \\
\hline CYP1D1 & ENSDARG00000035569 & ENSDART00000051565 & ENSDARP00000051564 & 5 & 23578519 & 23593172 & -1 & 7 & [59] \\
\hline CYP2AA1 & ENSDARG00000070017 & ENSDART00000102388 & ENSDARP000000093164 & 23 & 39112545 & 39130047 & 1 & 9 & \\
\hline CYP2AA2 & ENSDARG000000002981 & ENSDART00000006065 & ENSDARP00000021614 & 23 & 39132514 & 39145108 & 1 & 9 & \\
\hline CYP2AA3 & ENSDARG000000034070 & ENSDART000000012284 & ENSDARP000000014763 & 23 & 39160334 & 39172075 & 1 & 9 & \\
\hline CYP2AA4 & ENSDARG000000002981 & ENSDART00000045049 & ENSDARP00000045048 & 23 & 39040573 & 39050888 & 1 & 9 & \\
\hline$\overline{\text { CYP2AA6 }}$ & RNASEQDARG00000009292 & RNASEQDART00000009292 & $N / A^{b}$ & 23 & 38994308 & 39009052 & 1 & 9 & \\
\hline CYP2AA7 & ENSDARG00000002981 & ENSDART00000007810 & ENSDARP00000017742 & 23 & 39014695 & 39036855 & 1 & 9 & \\
\hline CYP2AA8 & ENSDARG000000002981 & ENSDART000000102420 & ENSDARP000000093197 & 23 & 39075131 & 39083899 & 1 & 9 & \\
\hline CYP2AA9 & ENSDARG000000070020 & ENSDART00000020538 & ENSDARP000000014406 & 23 & 39092849 & 39106493 & 1 & 9 & \\
\hline CYP2AA11 & ENSDARG000000041418 & ENSDART000000060715 & ENSDARP000000060714 & 23 & 39025380 & 39036455 & 1 & 9 & \\
\hline CYP2AA12 & ENSDARG000000002981 & ENSDART000000102932 & ENSDARP000000093707 & 23 & 38971320 & 38984900 & 1 & 9 & \\
\hline CYP2AA13 & & not in Ensembl Build 58 & & & & & & & \\
\hline CYP2AD2 & ENSDARG00000021172 & ENSDART00000024350 & ENSDARP00000018819 & 20 & 25174055 & 25181567 & -1 & 9 & \\
\hline CYP2AD3 & ENSDARG000000022650 & ENSDART00000063081 & ENSDARP000000063080 & 20 & 25187810 & 25191060 & -1 & 9 & \\
\hline CYP2AD6 & ENSDARG000000042956 & ENSDART00000063064 & ENSDARP000000063063 & 20 & 25165262 & 25173123 & -1 & 9 & \\
\hline CYP2AE1 & ENSDARG00000013524 & ENSDART00000035654 & ENSDARP000000030778 & 23 & 39385086 & 39398666 & 1 & 10 & \\
\hline CYP2AE2 & RNASEQDARG00000009297 & RNASEQDART00000009297 & NASEQDARP00000009294 & 23 & 39438355 & 39465937 & 1 & 10 & \\
\hline CYP2K6 & ENSDARG00000038371 & ENSDART00000055979 & ENSDARP00000055978 & 3 & 8561027 & 8571051 & -1 & 10 & [75] \\
\hline CYP2K7 & ENSDARG00000040433 & ENSDART00000101326 & ENSDARP000000992100 & 3 & 6855362 & 6872308 & -1 & 10 & \\
\hline CYP2K8 & ENSDARG000000040431 & ENSDART000000059182 & ENSDARP000000059181 & 3 & 8585106 & 8602953 & -1 & 9 & \\
\hline CYP2K16 & ENSDARG00000058458 & ENSDART000000081328 & ENSDARP000000075771 & 3 & 8606661 & 8622465 & -1 & 9 & \\
\hline CYP2K17 & ENSDARG00000038369 & ENSDART000000055971 & ENSDARP000000055970 & 3 & 8624449 & 8631739 & -1 & 9 & \\
\hline CYP2K18 & ENSDARG000000038366 & ENSDART00000055972 & ENSDARP000000055971 & 3 & 8671991 & 8677064 & -1 & 9 & \\
\hline CYP2K19 & ENSDARG00000038367 & ENSDART000000055973 & ENSDARP00000055972 & 3 & 8647105 & 8654962 & -1 & 9 & \\
\hline CYP2K20 & ENSDARG000000040426 & ENSDART000000059174 & ENSDARP000000059173 & 3 & 8657079 & 8669242 & -1 & 9 & \\
\hline CYP2K21 & ENSDARG000000040424 & ENSDART000000059171 & ENSDARP000000059170 & 3 & 8638383 & 8643321 & -1 & 9 & \\
\hline CYP2K22 & ENSDARG00000040433 & ENSDART000000015232 & ENSDARP00000012186 & 3 & 6809065 & 6872358 & -1 & 9 & \\
\hline CYP2K31 & ENSDARG00000009874 & ENSDART00000059185 & ENSDARP00000059184 & 3 & 6887113 & 6898613 & -1 & 9 & \\
\hline CYP2N13 & ENSDARG000000042953 & ENSDART000000063058 & ENSDARP000000063057 & 20 & 25154644 & 25162165 & -1 & 9 & \\
\hline CYP2P69 & ENSDARG00000042978 & ENSDART00000063100 & ENSDARP00000063099 & 20 & 25202489 & 25207822 & 1 & 9 & {$[90]^{9}$} \\
\hline
\end{tabular}


Table 2: Zebrafish CYPs in families 1-4, with Chromosomal location (Zv8), Ensembl gene ID (Release 58), and exon count (Continued)

\begin{tabular}{|c|c|c|c|c|c|c|c|c|c|}
\hline$\overline{C Y P 2 P 7}$ & ENSDARG00000042980 & ENSDART00000063107 & ENSDARP000000063106 & 20 & 25208167 & 25213192 & 1 & 9 & \\
\hline CYP2P8 & ENSDARG00000042982 & ENSDART00000063108 & ENSDARP000000063107 & 20 & 25214780 & 25219066 & 1 & 9 & \\
\hline CYP2P9 & ENSDARG00000022631 & ENSDART00000030229 & ENSDARP00000028831 & 20 & 25221011 & 25224248 & 1 & 9 & \\
\hline CYP2P10 & ENSDARG00000042990 & ENSDART00000063122 & ENSDARP00000063121 & 20 & 25225641 & 25232985 & 1 & 9 & \\
\hline CYP2P14 & ENSDARG00000042994 & ENSDART00000063128 & ENSDARP000000063127 & 20 & 25250397 & 25255115 & 1 & 8 & \\
\hline CYP2R1 & ENSDARG00000056587 & ENSDART00000079091 & ENSDARP000000073546 & 7 & 27793618 & 27799151 & 1 & 5 & \\
\hline CYP2U1 & ENSDARG00000026548 & ENSDART00000048281 & ENSDARP00000048280 & 1 & 49893260 & 49901816 & -1 & 6 & \\
\hline CYP2V1 & ENSDARG00000018485 & ENSDART00000016501 & ENSDARP00000011215 & 20 & 25191510 & 25197554 & 1 & 9 & \\
\hline CYP2X6 & ENSDARG00000079653 & ENSDART00000064591 & ENSDARP00000064590 & 25 & 13917523 & 13924246 & 1 & 9 & \\
\hline CYP2X7 & ENSDARG00000044002 & ENSDART00000064586 & ENSDARP00000064585 & 25 & 13906315 & 13912802 & 1 & 9 & \\
\hline CYP2X8 & ENSDARG00000043997 & ENSDART00000064596 & ENSDARP000000064595 & 25 & 13889786 & 13898258 & 1 & 9 & \\
\hline CYP2X9 & ENSDARG00000070775 & ENSDART00000104216 & ENSDARP000000094991 & 25 & 13881532 & 13888365 & 1 & 9 & \\
\hline CYP2X10C1 & ENSDARG00000006501 & ENSDART00000052054 & ENSDARP000000052053 & 7 & 52828535 & 52831744 & -1 & 9 & \\
\hline CYP2X10c2 & ENSDARG00000068283 & ENSDART00000098690 & ENSDARP000000089461 & 7 & 52971112 & 52974318 & -1 & 9 & \\
\hline CYP2X11 & ENSDARG00000068287 & ENSDART00000098703 & ENSDARP00000089474 & 7 & 53000061 & 53012216 & 1 & 9 & \\
\hline CYP2X12C1 & ENSDARG00000068290 & ENSDART00000098705 & ENSDARP00000089476 & 7 & 53013611 & 53022923 & 1 & 9 & \\
\hline CYP2X12C2 & ENSDARG00000068414 & ENSDART00000098924 ${ }^{d}$ & ENSDARP000000089694 & 7 & 54082352 & 54244690 & -1 & 10 & \\
\hline CYP2Y3 & ENSDARG00000068493 & ENSDART00000099074 & ENSDARP00000089848 & 15 & 59576 & 64111 & -1 & 9 & \\
\hline CYP2Y4 & ENSDARG00000007173 & ENSDART00000002842 & ENSDARP00000013280 & 15 & 70518 & 76649 & -1 & 9 & \\
\hline CYP3A65 & ENSDARG00000045627 & ENSDART00000067097 & ENSDARP000000067096 & 1 & 59169705 & 59179378 & -1 & 13 & {$[80]$} \\
\hline CYP3C1 & ENSDARG00000015575 & ENSDART00000018676 & ENSDARP00000009507 & 3 & 38092409 & 38099696 & -1 & 13 & {$[79$} \\
\hline CYP3C2 & ENSDARG00000037874 & ENSDART00000055203 & ENSDARP00000055202 & 3 & 38082058 & 38088687 & -1 & 13 & \\
\hline CYP3C3 & ENSDARG00000037873 & ENSDART00000102393 & ENSDARP00000093169 & 3 & 38071126 & 38078946 & -1 & 13 & \\
\hline CYP3C4 & ENSDARG00000070021 & ENSDART00000102416 & ENSDARP00000093193 & 3 & 38059261 & 38068888 & -1 & 13 & \\
\hline CYP4F43 & ENSDARG00000053530 & ENSDART00000063442 & ENSDARP000000063441 & 12 & 46414253 & 46432708 & 1 & 12 & \\
\hline CYP4T8 & ENSDARG00000004964 & ENSDART00000076955 & ENSDARP00000071423 & 16 & 3042131 & 3063539 & -1 & 12 & \\
\hline CYP4V7 & ENSDARG00000061585 & ENSDART00000087976 & ENSDARP00000082409 & Zv8 NA9570 ${ }^{f}$ & 11333 & 19450 & 1 & 11 & \\
\hline CYP4V8 & ENSDARG00000062132 & ENSDART00000089480 & ENSDARP000000083913 & 1 & 17310768 & 17323080 & 1 & 11 & \\
\hline
\end{tabular}

${ }^{a}$ GeneWise reprediction; ${ }^{b}$ no Ensembl gene prediction; ${ }^{c}$ identical DNA; ${ }^{d}$ identical DNA; ${ }^{e}$ incorrect N-term exon; ${ }^{\mathrm{f}} \mathrm{Chr} 1631205762$ is probably best location; ${ }^{\mathrm{a}}$ There are non-mammalian genes that share synteny but not the same subfamily name with mammalian CYP2J2 s. At present, annotation of fish CYP2 s by BLAST can result in misclassifying fish CYP2 s as CYP2Js (Nelson, 2005, Wang et al. 2007), as strictly interpreted according to nomenclature rules, there is not a CYP2J subfamily in fish. 
Table 3 Synteny comparison between zebrafish and human CYPs

\begin{tabular}{|c|c|}
\hline Zebrafish & Human \\
\hline CYP1A & CYP1A1/1A2 \\
\hline CYP1B1 & CYP1B1 \\
\hline CYP1C1,2 & - \\
\hline CYP1D1 & CYP1D1P \\
\hline CYP2Ks & CYP2W1 \\
\hline CYP2N13 & CYP2J2 \\
\hline CYP2Ps & CYP2J2 \\
\hline CYP2R1 & CYP2R1 \\
\hline CYP2U1 & CYP2U1 \\
\hline CYP2V1 & CYP2J2 \\
\hline CYP2X1-10 & - \\
\hline CYP2Y3,4 & CYP2A/B/F/S \\
\hline CYP2AA1-12 & - \\
\hline CYP2AD2,3,6 & CYP2J2 \\
\hline CYP2AE1,2 & - \\
\hline CYP3A65 & CYP3A-se1,-se $2^{a}$ \\
\hline CYP3С 1-4 & CYP3A3,4,7 \\
\hline CYP4F43 & CYP4F \\
\hline CYP4V7,8 & CYP4V2 \\
\hline CYP4T8 & - \\
\hline CYP5A1 & CYP5A1 \\
\hline CYP7A1 & CYP7A1 \\
\hline CYP7B1 & CYP7B1 \\
\hline CYP7C1 & - \\
\hline CYP8A1 & CYP8A1 \\
\hline CYP8B1-3 & CYP8B1 \\
\hline CYP11A1,2 & CYP11A1 \\
\hline CYP11C1 & - \\
\hline CYP17A1,2 & CYP17A1 \\
\hline CYP19A1,2 & CYP19A1 \\
\hline CYP20A1 & CYP20A1 \\
\hline CYP21A1 & CYP21A2 \\
\hline CYP24A1 & CYP24A1 \\
\hline CYP26A1 & CYP26A1/C1 \\
\hline CYP26B1 & CYP26B1 \\
\hline CYP26C1 & - \\
\hline CYP27A3-7 & CYP27A1 \\
\hline CYP27B1 & - \\
\hline CYP27C1 & - \\
\hline CYP39A1 & CYP39A1 \\
\hline CYP46A1 & CYP46A1 \\
\hline CYP46A2,4,5 & - \\
\hline CYP51A1 & CYP51A1 \\
\hline
\end{tabular}

${ }^{a}$ pseudogene (single remnant exon)

defects (cell migration defects). Zebrafish CYP11A2 is 80\% identical to CYP11A1 at the amino acid level, but little is known about functional similarities between the two forms, or about CYP11A2 expression, although based on our microarray results (see below) it appears to be expressed in the early embryo, and database EST evidence indicates that it is also expressed in adult gonads.

Zebrafish CYP11C1 exhibits $42 \%$ amino acid identity to human CYP11B2 (aldosterone synthase), but does not share synteny with any tetrapod CYP11B genes.

\section{CYP17 (steroid 17 $\alpha$-hydroxylase/17,20-lyase)}

CYP17 enzymes catalyze dual functions of steroid-17 $\alpha$ hydroxylase and steroid-17, 20-lyase. The zebrafish genome has two CYP17A genes that are $49 \%$ identical at the amino acid level, and the two genes are located on different chromosomes (Chr 13 and 23). One of these (CYP17A1) was cloned from zebrafish ovary [29]. CYP17A1 is expressed in gonadal tissue, but also in brain, gill, liver, and intestine. Zebrafish CYP17A1 is upregulated by fluorotelomer alcohols [30], but not by benzo[a]pyrene [31]. The functions of the two CYP17 s may differ (see Discussion, below) [32,33].

\section{CYP19 (aromatase)}

Zebrafish have two distinct CYP19 (aromatase) genes, CYP19A1 and CYP19B1 reported initially by Kishida and Callard [34]. The two aromatase forms are expressed in different tissues; CYP19A1 is expressed principally in the ovary, but also at much lower levels in the testis, while CYP19B1 is expressed in brain and neural tissue, including in the olfactory bulb, ventral telencephalon, preoptic area, and ventral/caudal hypothalamic zone, and in the anterior and posterior lobes of the pituitary [35]. Estrogen-mediated upregulation has been observed only for the neural form, CYP19B1, in adults and in embryos after $24 \mathrm{hpf}[34,36]$.

\section{CYP20A1 (undetermined function)}

Zebrafish have one CYP20 gene, CYP20A1, 61\% identical to the CYP20A1 in humans, and they share synteny (Table 3), both being adjacent to $A B I 2$. At present, the substrate specificity and biological function of CYP20A1 are unknown, and it remains an "orphan" CYP [37]. However, there is evidence for high levels of mRNA expression in the hippocampus and substantia nigra of mouse [38], suggesting functions in these parts of the brain implicated in important neuropathies.

\section{CYP21 (steroid 21-hydroxylase)}

Zebrafish CYP21A1 shares 39\% identity with human CYP21A2, and shares synteny with the human CYP21A gene pair (human CYP21A1P is a pseudogene). Mammalian CYP21A1 and CYP21A2 convert progesterone to deoxycorticosterone via 21-hydroxylation, prominently in the adrenal gland. The zebrafish CYP21A1 presumably serves a similar function, in the interrenal.

\section{CYP24 (Vitamin D3 metabolism)}

CYP24A1 is a mitochondrial enzyme catalyzing $1 \alpha$, 25-dihydroxyvitamin-D3-24-hydroxylase. There is one CYP24A1 gene in zebrafish, with $63 \%$ percent amino acid identity to human CYP24A1. The zebrafish enzyme is presumed to function similarly to mammalian 
CYP24 s, as vitamin D3 and various metabolites circulate in fish serum [39] and zebrafish have a functional vitamin D receptor (VDR; NR1I1) with ligand specificity similar to other vertebrate VDRs [40].

\section{CYP26 (retinoic acid hydroxylase)}

There are three CYP26 s in zebrafish. CYP26 was first discovered in zebrafish [41] and the retinoic acid metabolizing function appears to be conserved throughout the vertebrates [42-44]. Each zebrafish CYP26 is located on a separate chromosome, suggesting early establishment of these duplicated genes. The CYP26 s are categorized into three separate subfamilies, with some confusion as to the proper classification, i.e., zebrafish $C Y P 26 C 1$ has previously been referred to as $C Y P 26 D 1$ [45]. CYP26A1 and CYP26B1 share synteny with human $C Y P 26 A 1$ and $26 B 1$ genes, respectively, while the CYP26C1 syntenic relationship is less distinct (Table 3 ). The three zebrafish CYP26 $s$ are all induced by and metabolize retinoic acid, and play essential roles in hindbrain patterning [13,44-46]. Zebrafish CYP26 s also play roles in osteogenesis $[2,47]$, in pancreatic development [48], and presumably other processes.

\section{CYP27 (vitamin D3 metabolism)}

Zebrafish have seven distinct CYP27 genes, distributed into three subfamilies, while in most mammals there are two or three CYP27 genes. Human CYP27A1 and CYP27B1 catalyze vitamin D3 25-hydroxylase and 25hydroxyvitamin D3 $1 \alpha$-hydroxylase, respectively [22]. The five zebrafish $C Y P 27 A$ genes are all tandemly located on Chromosome 9 and share synteny with human CYP27A1 (Table 1). Zebrafish CYP27B1 and $C Y P 27 C 1$ are located on different chromosomes (Chr 11 and 6, respectively; Table 1). Functional properties of the various zebrafish CYP27 s are unknown.

\section{CYP39 (24-OH-cholesterol-7 $\alpha$-hydroxylase)}

A CYP39A1 is present in the zebrafish genome, located on chromosome 20. In mammals CYP39A1 functions as an oxysterol- $7 \alpha$-hydroxylase, involved in the conversion of cholesterol to bile acids. CYP39A 1 has not been found in other published fish genomes, but is present in tetrapod genomes, with conserved synteny to human CYP39A1. Zebrafish CYP39A1 is approximately $4 \mathrm{Mb}$ from a cluster of genes that share synteny with human CYP39A1.

\section{CYP46 (cholesterol 24-hydroxylase)}

Zebrafish have four CYP46A genes. One (CYP46A1) is on Chromosome 20, two (CYP46A2 and CYP46A4) are arranged in tandem on Chromosome 5. A fourth (CYP46A5) is supported by EST data but missing from the current assembly (Zv8). In contrast, humans have one CYP46, CYP46A1. Human CYP46A1 functions both as a cholesterol 24(S)-hydroxylase and as a 24-hydroxycholesterol-hydroxylase $[49,50]$. CYP46A1 in mammals is a brain-specific cholesterol-metabolizing enzyme, important for maintaining brain cholesterol homeostasis and membrane function, but also with potential regulatory roles as a neurosteroid-metabolizing enzyme [51].

\section{CYP51 (lanosterol 14 $\alpha$-demethylase)}

Zebrafish possess one CYP51 gene, CYP51A1. In other animals CYP51 is a lanosterol-demethylase enzyme, the only CYP involved in the post-squalene portion of the cholesterol biosynthesis pathway. CYP51 is an essential enzyme in the de novo synthesis of cholesterol [52]). CYP51 is often considered to be a "housekeeping gene" based on its ubiquitous expression and conserved function [53]. Zebrafish CYP51 has been cloned and heterologously expressed in E. coli [54]. Microarray analysis and in situ hybridization of embryonic zebrafish showed that CYP51 is strongly expressed in the eye, in the brain, and in epidermal cells of the forehead and the tail fin [55], and suggesting a role for CYP51 and other isoprenoid synthesis enzymes in hematopoietic and vascular development.

\section{CYP Families 1-4 (Xenobiotic Metabolizing CYPs)}

CYP families 1, 2, 3, and 4 include enzymes involved in drug, xenobiotic and fatty acid metabolism. In contrast to the CYPs that have principally endogenous functions, these CYP genes are much more diverse, with much less conservation of sequence between zebrafish and human. Table 2 provides specific information on the CYP 1-4 family genes. A molecular phylogeny of Clan 2 genes, which includes families 1 and 2 as well as CYP17 and CYP21, is shown in Figure 2. Molecular phylogeny of the Clan 3 genes, including the CYP3 s and CYP5 s (discussed above) and Clan 4 genes (the CYP4s) can be seen in Figure 1. Some relationships between zebrafish and human CYPs in families 1-3 suggested by molecular phylogeny have been substantiated by synteny analysis.

Clan 2- CYP1s

Zebrafish have five $C Y P 1$ genes in four subfamilies, $C Y P 1 A, C Y P 1 B 1, C Y P 1 C 1, C Y P 1 C 2$, and $C Y P 1 D 1$, all of which have been cloned and sequenced [56-59]. Zebrafish $C Y P 1 A$ has exon structures similar to the human $C Y P 1 A 1$ and $C Y P 1 A 2$, and the single $C Y P 1 B 1$ gene has a gene structure very similar to human $C Y P 1 B 1$. The CYP1Cs are closely linked single-exon genes $[57,60]$. The recently identified zebrafish $C Y P 1 D 1$ has a gene structure identical to zebrafish $C Y P 1 A$, and quite different from the $C Y P 1 B 1$ and $C Y P 1 C s$ [59]. Members of all four CYP1 subfamilies have been identified in other fishes and non-mammalian tetrapods, and the evolution of the CYP1 family has been discussed elsewhere in some detail $[59,60]$. Notably, the CYP1Cs are absent and $C Y P 1 D 1$ is a pseudogene in human and some other mammals [59,61]. CYP1A, CYP1B1 and the CYP1Cs are inducible by AHR agonists [58,62]. Vertebrate CYP1As and CYP1B1 generally are involved in the metabolism of 


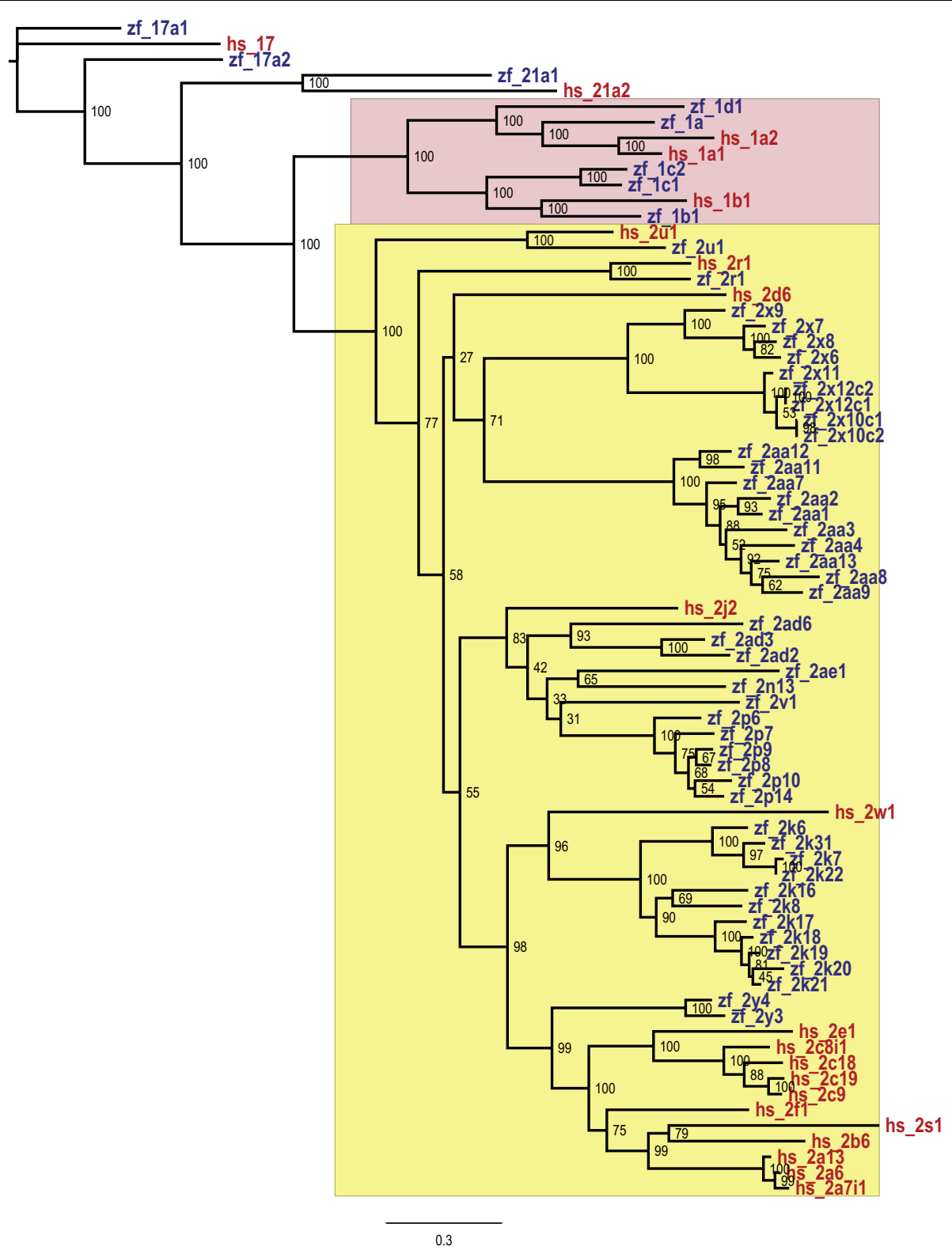

Figure 2 Maximum likelihood phylogenetic tree of zebrafish and human Clan 2 amino acid sequences. CYP1 family sequences are highlighted in red, while CYP2 sequences are highlighted in yellow. CYP2R1 and CYP2U1 are conserved. Zebrafish CYP2J co-orthologs clearly cluster with the human CYP2J, as do the zebrafish CYP2Ks with human CYP2W1, and zebrafish CYP2Ys with most of the remaining human CYP2 s. CYP1 evolution has been described elsewhere [58,59]. Human (hs) sequences are red, zebrafish (zf) are blue.

various drugs, many hydrocarbons, steroids and fatty acids. With the exception of benzo[a]pyrene and estradiol [63,64], little is known of the possible xenobiotic and endogenous substrates of the CYP1Cs and of CYP1D1.

\section{Clan 2 - CYP2s}

As in mammals, the CYP2 s constitute the largest CYP gene family in zebrafish, with 47 CYP2 genes, in contrast to 16 in humans. The subfamilies are considered below, in order according to suggested relationships: (i) orthology indicated by sequence, (ii) homologs suggested by sequence and orthology confirmed by shared synteny, and (iii) no evident homologous relationship to human CYPs.

(i) CYP2R and CYP2U There are single genes in each of these two subfamilies in zebrafish, CYP2R1 and $C Y P 2 U 1$. These can be classified as orthologs of human CYP2R1 and CYP2U1, based on sequence identity. They also exhibit shared synteny with their respective human counterparts (Table 3). CYP2R1 is a microsomal vitamin 
D 25-hydroxylase in humans [65,66]. Human CYP2U1 is expressed in the brain and the thymus [67], and catalyzes $\omega$ and $\omega-1$ hydroxylation of fatty acids, including arachidonic acid [68]. CYP2U1 appears to have a prevertebrate origin, and may be the oldest identifiable vertebrate CYP subfamily [69].

(ii) CYP2N, CYP2P, CYP2V, CYP2AD The 11 zebrafish genes in these five subfamilies occur in a clade together with human CYP2J2 (Figure 2). Analysis of gene location showed that the six CYP2Ps and CYP2N13, $C Y P 2 V 1, C Y P 2 A D 2, C Y P 2 A D 3$, and $C Y P 2 A D 6$ all are arranged in tandem on chromosome 20. This cluster of CYP genes shares synteny with human CYP2J2, indicating that all 11 zebrafish genes at this locus share an ancestral origin with CYP2J2 (Figure 3). CYP2J2 is an arachidonic acid epoxygenase with roles in the cardiovascular system. Functions of the members of the zebrafish cluster are unknown, although related genes in killifish (CYP2P3 and CYP2N1 and 2N2) are arachidonic acid hydroxylases and CYP2P3 in particular is similar in function to human CYP2J2 [70,71].

CYP2K Eight $C Y P 2 K$ genes are present in a tandemly duplicated array on Chromosome 3. This cluster of CYP2Ks shares synteny with human CYP2W1 (Additional File 1, Figure S2). Human CYP2W1 is a tumor-specific CYP [72,73] that oxidizes indole and chlorzoxazone, but not fatty acids [74]. CYP $2 K 6$, which is not highly expressed until $5 \mathrm{dpf}$, has been heterologously expressed, and demonstrated to catalyze the activation of the mycotoxin aflatoxin B1 (AFB1) to the carcinogenic exo-8,9AFB1 epoxide [75]. The low level CYP2K6 expression during zebrafish embryo and larval stages may help to explain the lack of AFB1 toxicity in zebrafish embryos [75]. Functions of the other CYP2Ks are not known.
CYP2Y CYP2Y3 and CYP2Y4 are tandemly arranged on chromosome 15 . They share synteny with a cluster of $C Y P 2$ genes for human drug-metabolizing enzymes, including CYP2A6, CYP2A13, CYP2B6, CYP2F1, and $C Y P 2 S 1$ located on human chromosome 19 (Additional File 1, Figure S3). The human genes at this locus include genes induced by PXR agonists (CYP2B6) and genes induced by AHR agonists (CYP2S1). Whether CYP2Y3 and CYP2Y4 have functional or regulatory properties in common with any of these human CYPs is not known.

(iii) CYP2X There are nine CYP2X genes present in two separate arrays of tandemly duplicated genes, on Chromosomes 7 and 25. Two of the CYP2X genes (CYP2X10 and $C Y P 2 X 12$ ) (Table 2), have two exact copies in the current genome assembly (Zv8; Ensembl Build 58) likely as an artifact of the assembly process, so there is some uncertainty as to the exact number of CYP2Xs. The $C Y P 2 X$ genes do not appear to share synteny with any mammalian CYP. Catalytic activities of CYP2Xs in zebrafish are unknown, although $C Y P 2 X 1$ from channel catfish has been cloned [76] and heterologously expressed [77]. Aminopyrine and benzphetamine demethylase activities were observed, but no biological function has as yet been ascribed.

CYP2AA Due to overlapping gene predictions, the current genome assemblies and gene counts are not accurate in the region on chromosome 23 where the $C Y P 2 A A$ genes are located. Ten CYP2AA genes are present in a tandemly duplicated array on chromosome 23. GeneWise [78] predictions indicate that there are 10 CYP2AAs, with high identity to each other (65-85\%), but no clear orthology to other CYP2 s. At least three have been cloned (CYP2AA1-2AA3; Bainy et al., Buhler et al.,

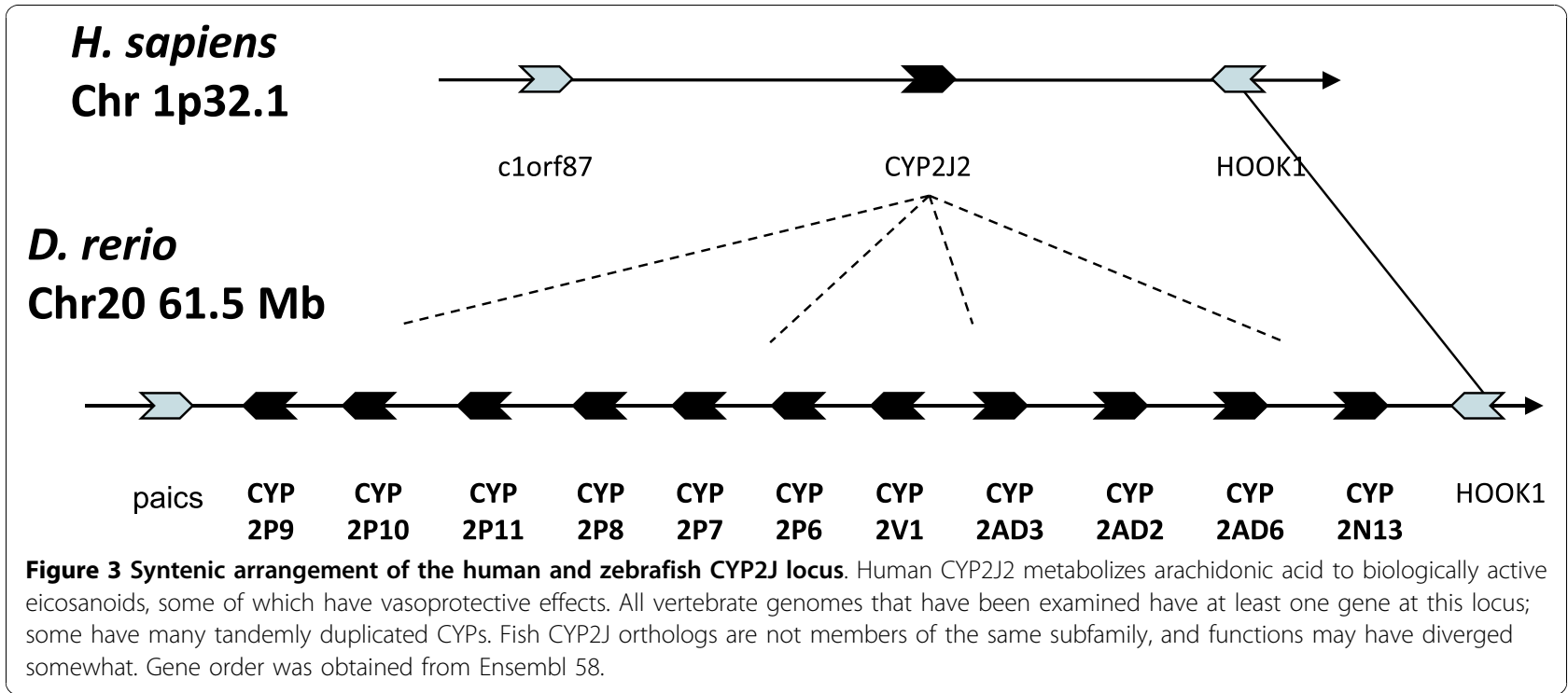


unpublished data). Some $C Y P 2 A A$ genes appear to be induced by PXR agonists (Kubota et al., unpublished data). The CYP2 s most closely related to the CYP2AAs are the CYP2Xs, and CYP2R1 (Figure 2).

CYP2AE The two CYP2AE genes are present as tandem duplicates on Chromosome 23, approximately $230 \mathrm{~kb}$ downstream from the CYP $2 A A$ cluster. No shared synteny is evident with mammalian genomes, and is unclear even for other published fish genomes. However, the $C Y P 2 A E s$ cluster phylogenetically with the CYP2J orthologs $C Y P 2 N / P / V / A E$ (Figure 2), suggesting a possible insertion of a set of duplicated $C Y P 2 J$ orthologs.

\section{Clan 3 - CYP3s}

The zebrafish genome includes five $C Y P 3$ genes, CYP3A65, and four CYP3Cs, CYP3C1-3C4 (Table 2). $C Y P 3 A 65$ and $C Y P 3 C 1$ were previously cloned and heterologously expressed $[79,80]$. CYP3A65 is a13 exon gene located in chromosome 1 , and is $54 \%$ identical to human CYP3A4. Zebrafish CYP3C1-CYP3C4 are all 13exon genes located in tandem on zebrafish chromosome 3 (Table 1). Other fish species also have multiple CYP3 genes located in this region (e.g. four in stickleback and one in fugu), and chickens appear to have two homologs at that locus. It is likely that the previously named human pseudogenes CYP3A-se1 and CYP3A-se2 [21] are the orthologous remnants of these $C Y P 3 s$, as they are located in the same region (on chromosome 7q22.1) as the immediately adjacent FOXK1gene (Additional File 1, Figure S3). Qiu et al. (2008) found that in most vertebrates apparently intact (i.e. not obviously pseudogenized) CYP3 genes are located within one of two genomic regions, labeled CYP3HR1 and CYP3HR2 [81]. Zebrafish CYP3Cs are within CYP3HR1, while the human CYP3As are in CYP3HR2. Zebrafish CYP3A65 does not share synteny with CYP3 $\mathrm{s}$ in other fish for which genomic information is available (medaka, stickleback, fugu, or tetraodon). CYP3A65 is inducible by some PXR agonists [80,82]. Other fish CYP3As are prominent in catalyzing testosterone $6 \beta$-hydroxylase [83], and this is likely true for zebrafish CYP3A65 as well. Activities are not known for the other zebrafish CYP3 s.

\section{Clan 4 - CYP4s}

There are four CYP4 genes in zebrafish, CYP4F43, CYP4T8, CYP4V7, and CYP4V8 (Table 2), in contrast to humans, which have 12 CYP4 s. Mammalian CYP4F enzymes function as omega-hydroxylases of $\mathrm{C}_{16}-\mathrm{C}_{26}$ fatty acids, including eicosanoids such as leukotriene B4 $[84,85]$. The full range of substrates of CYP4V enzymes is not yet known, although human CYP4V2 is a selective omega-hydroxylase of saturated, medium-chain fatty acids [86]. The CYP4 s appear to have less involvement in xenobiotic metabolism than many CYP1 s, CYP2 s or CYP3 s, although some xenobiotics (e.g., phthalates and perfluorooctanoic acid) induce CYP4Ts possibly via peroxisome proliferator-activated receptor alpha (PPAR $\alpha)$ and gamma (PPAR $\gamma)$ [87,88]. Zebrafish $C Y P 4 F 43$ and the CYP4VS share synteny with their human counterparts, but zebrafish CYP4T8 does not occupy a shared syntenic position with other CYP4 s.

\section{Developmental Expression}

Using single-color Agilent custom whole-transcriptome microarrays, we analyzed the expression of $88 \mathrm{CYP}$ genes over the course of early development in zebrafish (plot, Figure 4; heat map of normalized values, Figure 5). Zebrafish embryos were staged and sampled from 3 hours post-fertilization (hpf) through $48 \mathrm{hpf}$, and four biological replicates were sampled and analyzed (see Methods). After normalization, probes exhibiting saturation or signals that were not above background were removed (10 and 82 probes, respectively), leaving 21801 probes for examination of expression patterns. None of the excluded probes were for CYPs, indicating that all 88 CYPs examined were expressed during development. Bayesian Estimation of Temporal Regulation (BETR; [89]) showed 15393 significant differentially expressed probes $(\mathrm{p}<0.01)$, similar to the results of a naïve ANOVA analysis. Of the 88 CYP genes examined, 22 were found not to have differential expression during development. All others had one or more probes that indicated significant differential expression between sampling times.

Expression patterns of a subset of $11 C Y P s$ were assessed using qPCR. Molecule counts were calculated by using plasmid-derived standard curves, and expression levels were normalized to ARNT2. We previously observed [62] that $A R N T 2$ has significantly less variability during development than $\beta$-actin, a conclusion supported here by the single-color microarray data (Additional File 1, Figure S4). Nine of the eleven CYPs examined with qPCR exhibited linear relationships between the ARNT2-normalized microarray data and the ARNT2-normalized qPCR molecule counts (Figure 6).

\section{Patterns of Expression}

Several zebrafish CYPs exhibited expression levels that varied by 10 fold or greater (from low to high point) during development. Among these are CYP2P6, CYP11A2, CYP26A1, CYP26B1, CYP2AA7-CYP2AA9, $C Y P 2 A A 12, C Y P 2 Y 3, C Y P 2 Y 4$, and $C Y P 20$ (Figure 4). Some had highest levels of expression at the earliest time point ( $3 \mathrm{hpf}$ ). Others showed a peak at intermediate times, and still others showed elevated expression at the latest time sampled ( $48 \mathrm{hpf}$ ). Previous investigators have observed similar variation in expression levels for some of these CYPs, including CYP2P6 [90], CYP11A1 [28], and the CYP26 s [13,45]. 

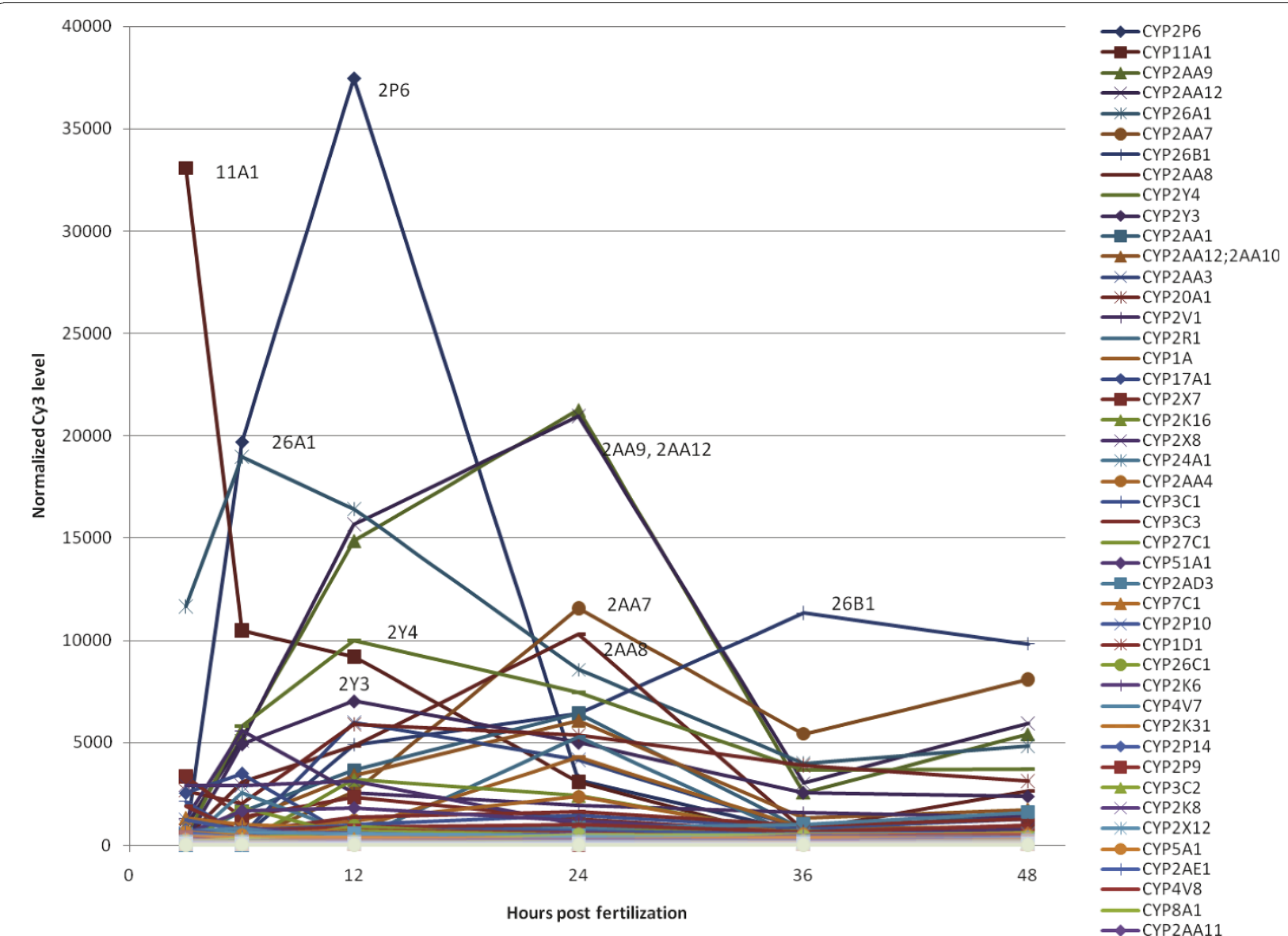

Figure 4 Expression of $\mathbf{8 8}$ CYP genes in zebrafish during the first $\mathbf{4 8}$ hours of development. Single color microarray analyses of CYP gene expression throughout early development (3-48 hours post fertilization, hpf) shows that while some CYP genes are expressed in the whole embryo at high levels, most CYP genes are expressed at levels significantly above background ( 5 fluorescent units; see Additional File 2 , Table S3). Strong temporal signals are apparent.

An alternate way of depicting developmental expression is a within-gene normalized heat-map, which shows the relative expression of all CYPs over the course of development (Figure 5). While the absolute expression levels of different genes may be low, especially in comparison to "housekeeping" genes such as $\beta$-actin or $A R N T 2$, within-gene normalization can reveal the relative changes in gene expression throughout the time series, and allow more ready comparison of profile clusters. This approach revealed a striking picture of waves of CYP gene expression as development progresses. The waves of CYP expression occurred with different clusters expressed most strongly usually only at one or two sequential time points (i.e., unimodally) during development. CYP2K22, CYP2N13, CYP3A65, and CYP8B1 appear to have bimodal expression, with elevated levels both at 3 and $48 \mathrm{hpf}$ (Figure 5). A similar bimodal expression pattern was seen with CYP5A1, CYP21A1, CYHP2K17, CYP8B3, and CYP4T8, which had the highest relative expression at $3 \mathrm{hpf}$ and $36 \mathrm{hpf}$.
Clustering of gene expression patterns (K-means clustering) revealed co-expression of tandemly duplicated CYPs, evident in the expression clustering of subsets of the CYP2AAs, the CYP2Ks, the CYP2Ys, the CYP46As, and the CYP27As (Figure 5). However, other clusters, such as the set of CYP2 subfamilies that share synteny with human CYP2J2 did not exhibit complete co-expression: CYP2P10, CYP2P14, CYP2AD2, and CYP2AD6 were mostly highly expressed at $3 \mathrm{hpf}$, while CYP2P6, CYP2P7, and CYP2V1 were most highly expressed at 6-12 hpf.

The observation that some CYPs showed high levels of transcript at $3 \mathrm{hpf}$, implies that these could involve maternally-derived transcript in oocytes. A number of CYP genes identified by cluster affinity search analysis using $\mathrm{MeV}[89,91]$ appear to have a maternal signal in the microarray data, based on the decreasing expression levels from $3 \mathrm{hpf}$ (Additional File 1, Figure S5 and Additional File 2, Table S1). To begin assessing maternal transcript we examined selected CYP in unfertilized 


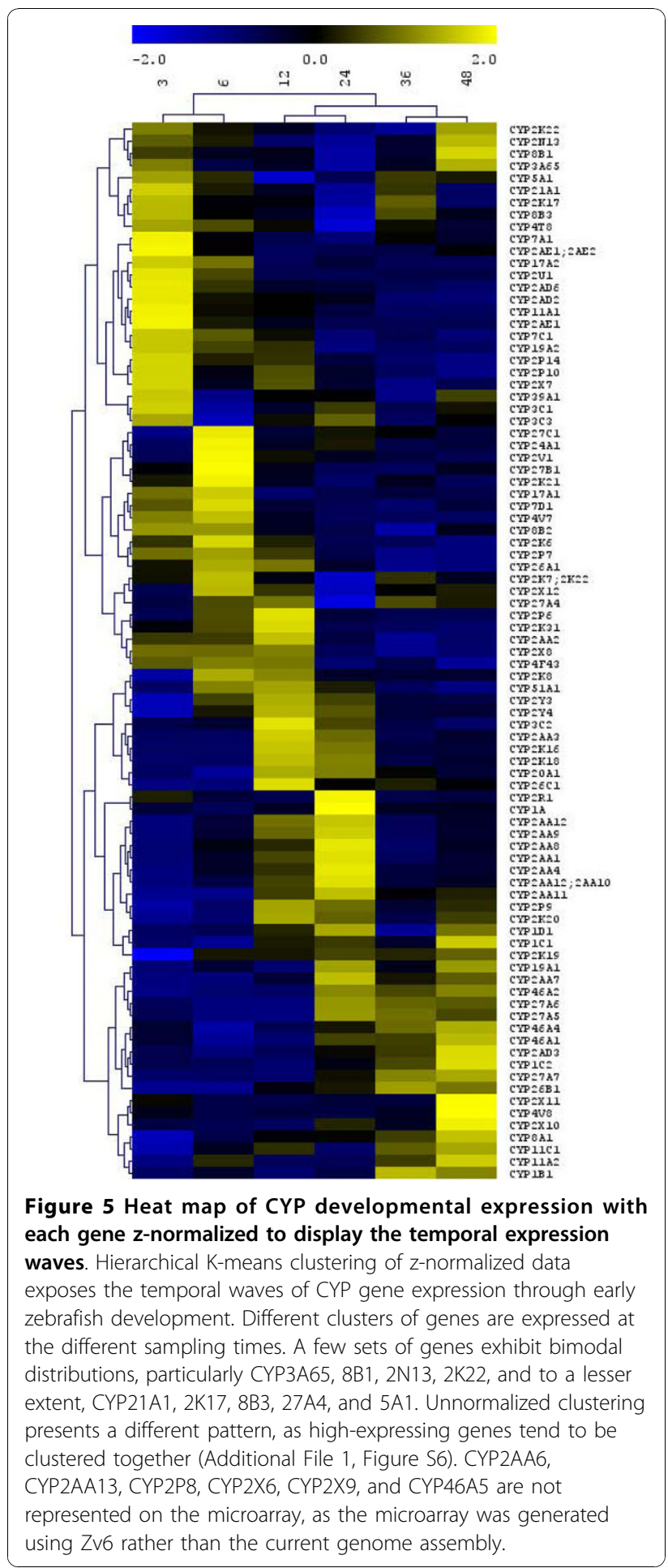

eggs. qPCR analysis of unfertilized eggs showed that $C Y P 1 A$, CYP2V1, CYP2AA4, and CYP20 had significant ARNT2-normalized expression levels in unfertilized eggs (Figure 7). Analysis of the larger complement of CYP genes for maternal transcript in unfertilized eggs requires microarray analysis, and is ongoing.

\section{Discussion}

In this study we identified the total CYP complement in zebrafish, and assessed patterns of expression of these genes during normal embryonic development. Zebrafish are an increasingly important model in developmental toxicology, pharmacology and chemical effects on disease. Knowing the identity and regulation of CYP genes is essential to strong inference regarding chemical effects in this model, and to assess pathways of metabolism of xenobiotics and endobiotics, and the relationship to CYP roles in these processes in humans. The $94 \mathrm{zeb}-$ rafish CYPs occur in the same 18 gene families that are found in humans and other mammals, but with differences in numbers of genes, and often with uncertain function.

\section{CYP Families 5-51}

Many of the CYP gene families in this group have single genes in zebrafish, as they do in humans, and show a high degree of conservation of sequence with their human counterparts. These genes also exhibit similar syntenic relationships as found in human. Together, the sequence data and gene location data indicate that in many of these families the genes are direct orthologs of their human (mammalian) counterparts. Where there is $1: 1$ correspondence in these gene families, i.e., with CYP5A1, 7A1, 7B1, 8A1, 20A1, 21A1, 24A1, 26A1, 26B1, 39A1, 46A1 and 51A1, it most likely indicates conservation of enzyme activities and physiological function. In other gene families in the "endogenous set", defining relationships between human CYPs with important endogenous functions and the zebrafish homologs is complicated by the presence of multiple closely related paralogs in zebrafish, not found in human. Thus, zebrafish have two CYP17As, two CYP19As, and four CYP46As, while humans have only half that number in each case. Such doubling of the numbers of genes in several CYP gene families could be the result of individual gene duplication, or could be remnants of the third round of whole genome duplication (WGD-3 [24,92]), with the retention of duplicated genes in zebrafish.

Zebrafish CYP paralogs that are co-orthologs of the human CYPs could have distinct functions, as a result of function partitioning (subfunctionalization) or differential regulation (temporal and/or organ differences or differences in induction). This has been observed for CYP19, with CYP19A1 (ovarian) and CYP19A2 (brain) aromatases displaying distinct expression patterns and inducibility. The neural form (CYP19A2) exhibits sensitivity to induction by estrogen, while the ovarian form appears to be mostly recalcitrant to induction by estrogen receptor agonists [36,93-95]. While functions have not been confirmed for zebrafish, the two CYP17A genes in other fish also appear to represent enzyme 

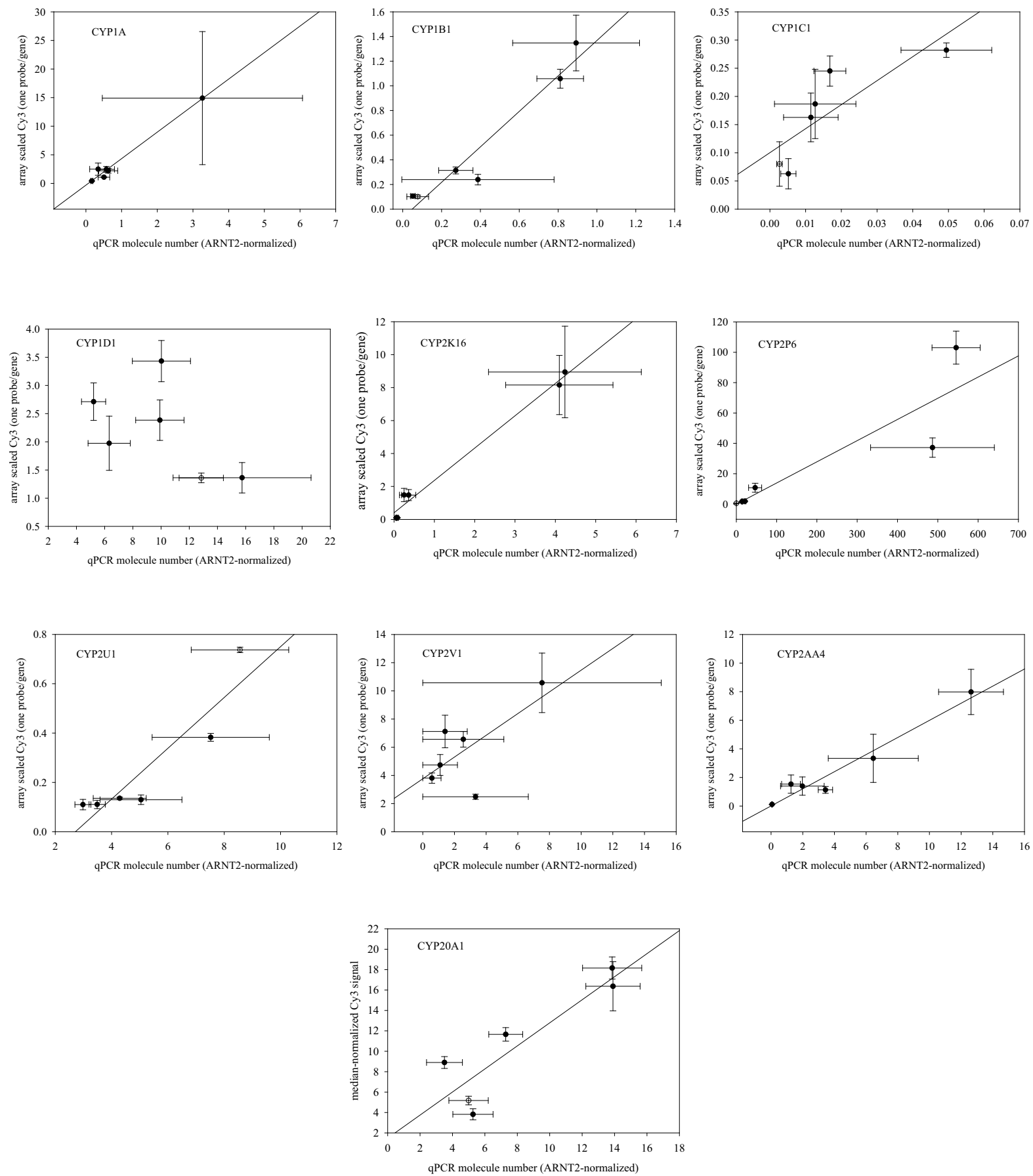

Figure 6 RT-qPCR analysis of CYP expression for $\mathbf{1 2}$ genes. CYP1A, 1B, 1C1, 1D1, 2K16, 2P6, 2U1, 2V1, 2AA4, 2AA6, 2AA11 and CYP20A1 were examined. qPCR confirms the microarray results for 10 of the 12 CYP genes examined. Both microarray and qPCR analyses were normalized by ARNT2 expression. Only CYP1D1 and CYP2AA4 exhibit deviations from linearity.

sub-functionalization following gene duplication. Tilapia and medaka CYP17A1 possess both steroid-17 $\alpha$-hydroxylase and 17, 20-lyase activities, as does mammalian CYP17A1, but both tilapia and medaka CYP17A2 possess only $17 \alpha$-hydroxylase activity, as they only convert pregnenolone or progesterone to $17 \alpha$ - hydroxy products, but do not perform the subsequent conversion to androstenedione or DHEA [32,33]. There also are significant differences in spatial expression patterns for the duplicated CYP17A genes during development, and 


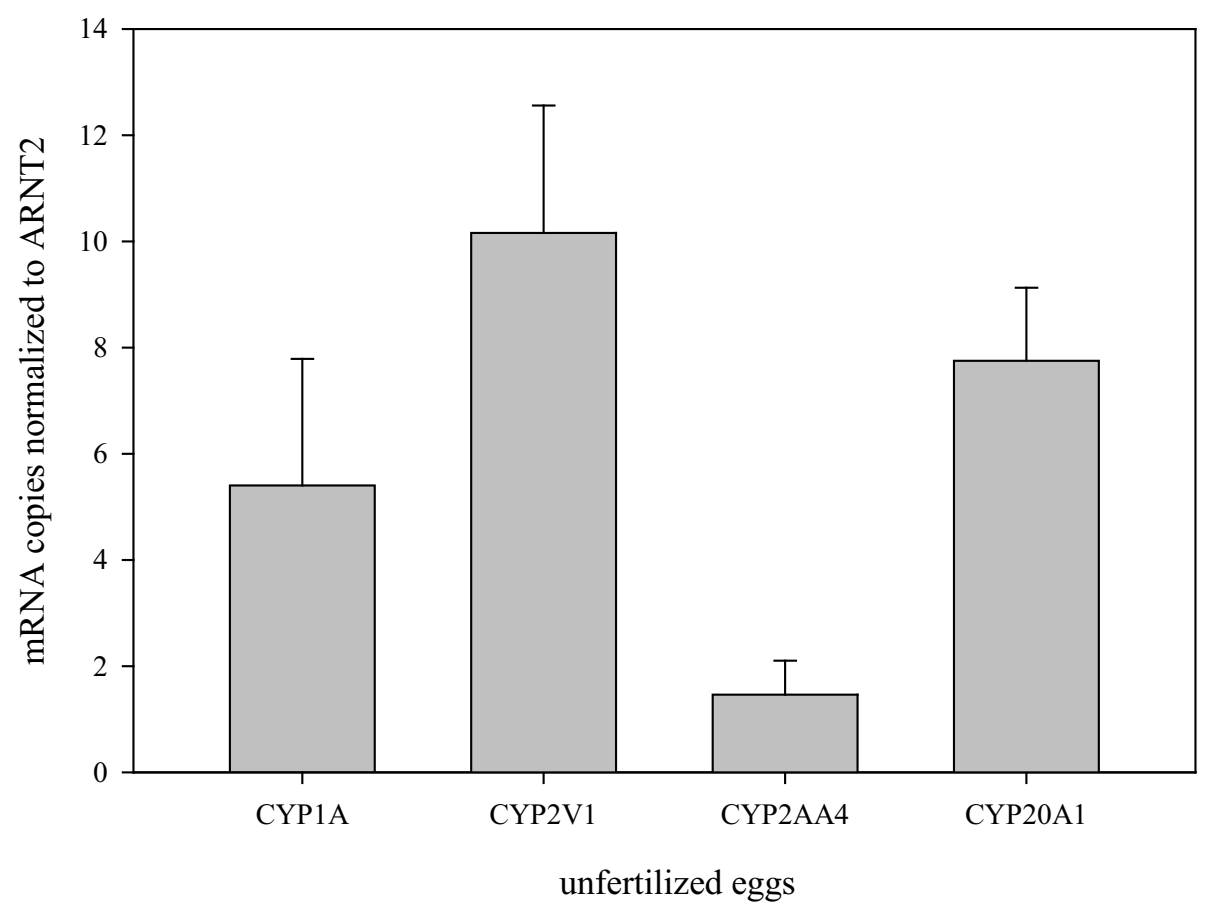

Figure 7 Expression of four CYP genes in unfertilized oocytes. The maternal contribution to transcript abundance of selected CYPs was determined using GPCR on unfertilized oocytes. Eggs were expressed from gravid female zebrafish $(n=3)$ by gentle squeezing of anesthetized fish. Data was normalized using ARNT2.

during the spawning cycle in other fish [32]. We did not see any substantial temporal separation of $C Y P 17 A 1$ and CYP17A2 expression during development, although CYP17A1 is more strongly expressed (Figure 4 and Additional File 2, Table S2).

In some families of "endogenous" genes, zebrafish have more than twice the number of genes than occur in humans, and some have novel subfamilies as well. This is evident in the CYP27s, and the CYP46s, where the numbers of genes are greater than would be expected to have resulted from WGD-3. It is likely that the ancestral condition would be one of fewer genes, with expansion in zebrafish rather than loss in humans. That expansion in zebrafish could involve WGD, but is most evident in tandem duplication as well as translocation. This is clearly suggested in CYP27 by the four genes that share synteny with the single human CYP27A1, and the presence of CYP27B1 and 27C1, which do not share synteny with human CYPs. The biological significance of some duplicated genes also could involve distinctions in temporal or organ- and cell-specific regulation, but determining this can be complicated by the strong possibility of substrate overlap.

\section{CYP Families 1-4}

Zebrafish CYP genes in families 1, 2, and 3 are more diverse than in humans, and sequence identities often are too low to discern orthology between zebrafish and mammalian genes in these families. However, analysis of the additional character of shared synteny clarifies evolutionary relationships between human and zebrafish genes in these families. CYP family 4 differs from families 1-3, as there are fewer CYP4 genes in zebrafish than there are in mammals. However, like the CYP1 s, CYP2 $\mathrm{s}$ and CYP3 s, the CYP4 s also are involved with (induced by or metabolize) xenobiotics, while this is seldom the case with CYP5-CYP51 genes.

Consistent with phylogeny, the fish CYP1 and CYP3 clades appear as sister groups to the mammalian clades for these genes. However, as discussed earlier [60], both mammals and fish share the CYP1 subfamilies CYP1A and CYP1B. Zebrafish also express CYP1Cs, which do not occur in humans, and CYP1D1, a pseudogene in human. All mammalian CYP3 $\mathrm{s}$ are in a single subfamily, CYP3A, which occurs in zebrafish as well. However, zebrafish CYP3A65 shares synteny with single exon pseudogene CYP3As in human (CYP3A-se1 and -se2), while the novel CYP3C subfamily [79] shares synteny with the functional human CYP3A3, 3A4 and 3A7 [81]. Fugu and possibly other fishes also have a second CYP3 subfamily, CYP3B, about which nothing is known $[96,97]$.

The functional similarities in different taxa, suggest similar biological roles for the homologous CYP1 $\mathrm{s}$ and 
CYP3 s. Thus, CYP3As are the primary catalysts of testosterone 6 $\beta$-hydroxylase in fish and mammals [98], and mammalian and fish CYP1As and likely CYP1Bs are prominent in the metabolism of some $\mathrm{PAH}$ pro-carcinogens. The roles of orthologous CYPs in metabolism of particular compounds can differ between taxa, however. Thus, the regio-specific oxidation of $\mathrm{BaP}$ and the rates of metabolism of planar HAH appear to differ in degree between fish and mammalian CYP1As [99,100], apparently reflecting species differences in CYP1A structures [101]. The functions of the novel CYP1 s and CYP3 s are less well defined, although zebrafish CYP1Cs and CYP1D1 have been expressed and functions have been determined with $\mathrm{BaP}$ [64], estradiol [63], and a number of other exogenous and endogenous substrates (Urban, Stegeman, et al. unpublished results). Little is known of the function of the CYP3Cs, but CYP3C1 appears not to be responsive to chemicals that induce CYP3A65 [79].

Identifying zebrafish-human orthologs is most difficult in the CYP2 family, where the differences in CYP2 divergence between zebrafish and humans obscure many homologous relationships. Thus as noted, of the 11 zebrafish and 11 human CYP2 subfamilies, only two (CYP2R and CYP2U) warrant the same designation in zebrafish as in humans based on sequence identity. While the disparity between zebrafish, or other fish, and mammals is exaggerated by evolutionary distance, our analysis of shared synteny indicates that members of distinct subfamilies in mammals and fish still may bear co-orthology. The CYP2J-related genes are a key example. The single clade of 11 genes in the zebrafish $C Y P 2 N, 2 P, 2 V$, and $2 A D$ subfamilies and human CYP2J2 (Figure 2), implies relationship between the fish and human genes. Our analysis shows the zebrafish genes occur in tandem in a cluster that shares synteny with CYP2J2, indicating co-orthology (Figure 3). This suggests that there are catalytic functions among these zebrafish CYPs that are similar to the human CYP2J2. A similar hypothesis was borne out in functional characterization of previously identified killifish CYP2P3, which also clusters with the CYP2Js on phylogenetic analysis. Heterologously expressed CYP2P3 exhibited nearly identical regio- and stereoselectivity for oxidation of arachidonic acid as human CYP2J2, consistent with molecular phylogeny indicating a shared ancestral origin [71]. Notably, at present there is little or nothing known about the catalytic or biological functions or the chemical regulation of the majority of zebrafish CYP2 s.

\section{Zebrafish CYPs in development}

In addition to annotating the full complement of CYP genes, we analyzed the expression of 88 CYP genes over the course of development in zebrafish (Figure 4). Several of these zebrafish CYPs exhibited markedly elevated expression levels at some time during development, but a large number, including more than three-quarters of the total genomic complement, showed distinct temporal expression patterns (Figure 5, Additional File 1, Figure S6). Importantly, gene expression profiling performed on whole embryos often under-estimates the importance of tissue- or cell-specific gene expression due to dilution effects.

Our array results are similar to developmental expression that has been determined for some individual CYP genes (e.g. CYP1 s [59,62], CYP2K6 [75], CYP3C1 [79], CYP19 [36,102]). Developmental roles have been established principally for some of the "endogenous" CYPs. Such genes include CYP11A1, which is essential for the synthesis of pregnenolone, critical for cell migration [27]; and the three CYP26 s, which contribute to retinoic acid gradients that regulate hindbrain and neural crest patterning $[13,103,104]$ and osteogenesis $[2,47]$. We and others have seen complex CYP expression patterns in development. Developmental roles of CYPs in families 1-3 are unknown, although morpholino knockdown of CYP1Cs appears to protect from developmental toxicity of dioxin (Kubota et al., unpublished data) and over-expression of CYP2P6 caused developmental abnormalities, including cardiovascular abnormalities [90], suggesting developmental significance of these genes.

The roles for many CYPs, including roles in development, cannot necessarily be inferred from sequence identities. Thus, CYP20A1 is an 'orphan' CYP that does not have a defined function in zebrafish or in humans $[37,38]$, and some large subfamilies (CYP2X, CYP2AA) do not have any homologs in mammals. As well, the different numbers of genes that are co-orthologs of single human CYPs precludes assignment of a function to any one, which requires empirical determination. This is true for the multiple co-orthologs in the "endogenous" CYP families, such as the CYP11 s, the CYP27 s and CYP46's, as well as for most those in the "xenobiotic" CYP families. The issues in CYP11 exemplify the questions and approaches. Zebrafish $C Y P 11 A 1$ is expressed throughout development, as in the murine model, but the CYP11A1 knockdown is not lethal $[5,27]$. As zebrafish have two $C Y P 11 A$ genes and a $C Y P 11 C$ gene, it is possible that overlapping substrate specificity and spatiotemporal expression patterns might allow one to substitute for the other in loss-of-function studies.

There is a greater dearth of information regarding CYP genes that may have maternally derived transcripts deposited in oocytes. Our analysis of transcripts of a few selected CYP genes, CYP1A, CYP2V1, CYP2AA4, and CYP20A1, showed that transcripts for all four were present in unfertilized zebrafish eggs, and that the levels of transcript could be substantial. CYP19 mRNA also has 
been reported in oocytes [105], and CYP1A transcript also was reportedly recently by others [106]. The significance of maternal transcript of these CYPs is not known. Whether other CYPs also have maternal transcripts deposited in oocytes, and what influences that deposition, is under investigation.

\section{Conclusions}

The identification of the full complement of 94 zebrafish CYPs, and determination that the majority of CYPs have distinct developmental patterns of expression opens the door to assessing their role(s) not only in development, but also in the response of embryos to toxic chemicals. For many CYP genes involved in xenobiotic metabolism, inferring relationships among the genes between zebrafish and human based on sequence is difficult, complicated by remnants of whole genome duplication in the teleost line, uneven gene duplication and gene loss in different animal and gene lineages, and the possibility of gene conversion of closely linked paralogs [107]. Nevertheless, shared synteny indicates orthologous relationships of many zebrafish and human CYPs, and also indicates which zebrafish CYPs have no apparent homologs in humans. The lineage-specific diversification makes possible the acquisition of new functions (subfunctionalization) and regulation. In most cases, however, functions of the individual paralogous CYPs in zebrafish have yet to be determined. Those functions, and the timing, location and magnitude of expression in development will determine the strength of inference from developmental toxicological studies with the zebrafish model.

\section{Methods}

\section{Gene identification and sequence analysis}

Cytochrome P450 genes were identified by hidden Markov model searches (HMMER v2.3.2; [108]) of the ENSEMBL Zv6 and Zv7 gene predictions, using the global PFAM model for cytochrome P450 s (p450-ls). Annotations were transferred to the Zv8 alignment when it became available. Alignments were constructed using Muscle v3.6b [109], and masked based on the Muscle alignment scoring function. Phylogenetic trees were constructed by analyzing inferred or confirmed amino acid sequences using maximum likelihood (RAxML 7.0.4, [110]). The WAG model of amino acid substitution [111] with a gamma distribution of substitution rates was used in all Bayesian analyses (WAG $+\mathrm{G})$. Synteny was examined using the ENSEMBL and UCSC browsers.

\section{Animals}

Tubingen long fin (TL) zebrafish were progeny of TLs obtained from the laboratory of Dr. Mark Fishman, crossed with TLs raised from eggs obtained from the Zebrafish International Resource Center at the University of Oregon (Eugene, OR, USA). Fish were maintained in the Woods Hole Oceanographic Institution Zebrafish Facility and the experimental procedures were approved by the Institutional Animal Care and Use Committee. The zebrafish were held in 2:1 female to male groups at a density of $\leq 5$ fish $/ 1$ in aerated, filtered and re-circulated system water $\left(28.5^{\circ} \mathrm{C}\right)$ in 3 or $10 \mathrm{l}$ tanks in an Aquatic Habitat ${ }^{\mathrm{tm}}$ system. The system water was composed of Instant Ocean ${ }^{\mathrm{mw}}(60 \mathrm{mg} / \mathrm{l})$, sodium bicarbonate $(50 \mathrm{mg} / \mathrm{l})$, calcium sulfate $(8.5 \mathrm{mg} / \mathrm{l})$ and Kent's Freshwater Essentials ${ }^{\text {rut }}(53 \mu \mathrm{l} / \mathrm{l})$ in distilled water. Fish were fed twice daily with brine shrimp (Artemia salina) and once daily with Omega One flakes (Omega Sea Ltd. Sitka, AK, USA).

\section{Zebrafish embryos}

TL embryos were obtained from group breedings of 30 female and 15 male fish. Fertilized TL zebrafish eggs (100) were placed in $20 \mathrm{~cm}$ glass Petri dishes containing $50 \mathrm{ml}$ of $0.3 \mathrm{x}$ Danieau's solution. The embryos were incubated at $28.5^{\circ} \mathrm{C}$. At $24 \mathrm{hpf}$, the solutions were replaced with fresh $0.3 x$ Danieau's solution and any dead embryos were removed; mortality was normally four or less per dish. For sampling, all embryos in a dish were pooled, frozen in liquid nitrogen, and stored at $-80^{\circ} \mathrm{C}$. Sampling times were $3,6,12,24,36$, and $48 \mathrm{hpf}$. Each of four dishes was maintained independently, as biological replicates.

A separate collection of unfertilized eggs was taken to determine the maternal contribution to transcript abundance of selected CYP genes, which could imply function in early development. Eggs were expressed from gravid female zebrafish $(\mathrm{n}=3)$ by gentle squeezing of anesthetized fish, following published protocols [112]. Eggs that showed development of a perivitelline space were considered mature eggs and were selected for analysis.

\section{Microarray}

The Agilent (Agilent Technologies, Santa Clara, CA) $4 \times$ 44k DNA gene expression microarray was used to probe developmental gene expression. The original Agilent gene set was missing many CYPs and other genes involved in chemical defense. Probes for these genes were custom designed using the Agilent eArray system and added to the array. The individual microarrays had 21893 unique probes (excluding controls) printed in duplicate.

Microarrays were used to examine gene expression levels for all four replicates of each timepoint $(3,6,12$, 24, 36, and $48 \mathrm{hpf}$ ). Total RNA was extracted using the Aurum Fatty and Fibrous Tissue kit (Bio-Rad, Hercules, 
CA). RNA samples were checked for quality using a NanoDrop ND-1000 spectrophotometer and an Agilent 2100 BioAnalyzer. For each RNA sample, a single microarray was hybridized with 750 ng Cy3 labeled cDNA using Agilent's standard conditions for singlecolor microarrays at the Whitehead Center for Microarray Technology. The Agilent Low-Input QuickAmp Labeling Kit was used for labeling, the samples were hybridized to a the custom Agilent $4 \times 44 \mathrm{~K}$ feature zebrafish microarray using the Agilent In situ Hybridization Kit Plus, and labeled cDNA was combined with the Agilent $10 \times$ Control Targets (to identify microarray corners). Post-hybridization, microarray slides were washed as per the Agilent In situ Hybridization Kit Plus. Arrays were scanned with an Agilent DNA Microarray Scanner.

\section{Microarray analysis}

Analysis of raw microarray results was performed using Agilent's Feature Extraction software with background detrending (spatial and multiplicative). The data were then normalized using the non-linear scaling method based on rank invariant probes of Schadt et al. $[113,114]$, commonly performed for Affymetrix microarray data by the dChip software [115] and performed for our Agilent data by software developed by AGM. Briefly, this method finds the microarray with the overall median Cy3 signal for use as a baseline microarray (here 12 hpf, replicate C) to which all other microarrays are normalized. For normalization, microarrays are each separately normalized to the baseline microarray by finding Rank Invariant probes between the two microarrays using the method outlined in Schadt et al. [113]. The software develops a non-linear normalization curve for each microarray and uses a piecewise linear running median to normalize the data. Prior to normalization any Cy3 values below 5 were set to 5 and after normalization the average normalized signal was calculated for probes duplicated on the microarray. Probes were removed from consideration when they exhibited saturation in any instance among the microarrays and duplicate probes, or when signals were not above background, in all instances among the microarrays and duplicate probes. Bayesian Estimation of Temporal Regulation (BETR; [89]) was used to analyze the developmental time series, as standard ANOVA is not appropriate due to auto-correlation between time points. Normalized Cy3 values for each probe were log transformed, median-centered, and analyzed using BETR relative to $3 \mathrm{hpf}$.

Gene expression clustering was analyzed using $\mathrm{MeV}$ (v4.3) [116]. Hierarchical K-means clustering of both un-normalized and within-gene $\mathrm{z}$-normalized mean gene expression values ( $\mathrm{n}=4$ biological replicates) was performed, assuming 10 clusters. A different measure of clusters was performed using the Cluster Affinity Search Technique [91], setting the cluster affinity to 0.7 (0-1 scale).

Microarray data has been deposited in the Gene Expression Omnibus (GEO) database (GEO accession number GSE24840).

\section{Real-time, quantitative RT-PCR}

RNA quantity and quality were determined spectrophotometrically (NanoDrop ND-1000; NanoDrop Technologies, Wilmington, DE, USA). cDNA was synthesized using the Omniscript ${ }^{\mathrm{Tm}}$ Reverse Transcriptase kit (Qiagen Inc., Valencia, CA, USA), random hexamer primers (Operon Biotechnologies Inc.) and the RNasin ${ }^{\bullet}$ RNase inhibitor (Promega). Gene-specific PCR primers for zebrafish $C Y P$, and $A R N T 2$ were synthesized by Operon Biotechnologies Inc (Table S1). (The ARNT2 primers were designed to amplify a sequence common to $A R N T 2 a, b$, and $c[58])$. Real time PCR was performed using the iQ SYBR Green Supermix (Bio-Rad) as previously described $[58,62]$. To ensure that a single product was amplified, melt curve analysis was performed on the PCR products, and polyacrylamide gel electrophoresis was performed after the PCR run for each gene. The specificity of the CYP qPCR primer pairs was tested by purifying and sequencing the cDNA products of the final qPCR reactions.

A standard curve for each gene was generated by serially diluting plasmids containing the $100-150$ bp region amplified by the quantitative PCR primers. Total molecule numbers were calculated for each sample and normalized to ARNT2 expression [58]. In the figures data are shown as mean + standard deviation of the mean (SD).

\section{Additional material}

Additional file 1: Additional Figures. Orthologous relationships between zebrafish and human CYPs, synteny analyses for human CYP2W1-zebrafish CYP2Ks and human CYP2ABFGST-zebrafish CYP2Ys, expression of control genes, and alternatively microarray expression clustering including CAST results and unnormalized hierarchical clustering.

Additional file 2: Additional Tables. CYP2J-like genes in zebrafish, CYP genes clustered by developmental expression patterns (CAST), singlecolor median-scaled Cy3 expression values for microarray probes, and qPCR primer sequences.

\section{Acknowledgements}

This work was supported by NIH grants R01ES015912 and P42ES007381 (Superfund Basic Research Program at Boston University) (to JJS). MEJ was a Guest Investigator at the Woods Hole Oceanographic Institution (WHOI) and was supported by grants from the Swedish research council Formas and Carl Trygger's foundation. AK was a Post-doctoral Fellow at WHOI, and was supported by a fellowship from the Japanese Society for Promotion of Science (JSPS). JZ and TP were Guest Students at the WHOI and were 
supported by a CAPES Ph.D. Fellowship and CNPq Ph.D. Sandwich Fellowship (JZ), and by a CNPq Ph.D. Fellowship (TP), from Brazil. The sponsors had no involvement in performing or in the decision to publish this study. The U.S. Government is authorized to produce and distribute reprints for governmental purposes notwithstanding any copyright notation that may appear hereon

\section{Author details}

${ }^{1}$ Biology Department, Woods Hole Oceanographic Institution, Woods Hole, MA, USA. ${ }^{2}$ Andrew McArthur Consulting, 11 Roanoke Road, Hamilton, Ontario, Canada. ${ }^{3}$ Instituto de Ciências Biológicas, Universidade Federal do Rio Grande, Rio Grande, RS 96201-900, Brazil. ${ }^{4}$ Instituto de Biofísica Carlos Chagas Filho, Universidade Federal do Rio de Janeiro, Rio de Janeiro, Brazil. ${ }^{5}$ Department of Environmental Toxicology, Uppsala University, Uppsala, Sweden. ${ }^{6}$ Department of Molecular Sciences, University of Tennessee, Memphis, TN, USA.

\section{Authors' contributions}

JVG helped conceive of the study, participated in its design and coordination, carried out the microarray experiments, phylogenetic and synteny analyses, literature review, assisted with the nomenclature, and drafted the manuscript. AMG analyzed the microarray data and performed the statistical analyses for microarray gene expression. AK performed qPCR on unfertilized eggs. JZ, TP, and MJ performed qPCR analyses for confirmation of the microarray results. DRN assisted in the sequence analysis and established the gene nomenclature. JJS conceived and designed the study, obtained support, coordinated its execution, and drafted the manuscript. All authors read, commented on, and approved the final manuscript.

Received: 26 August 2010 Accepted: 18 November 2010

Published: 18 November 2010

\section{References}

1. Shen $A L$, O'Leary KA, Kasper CB: Association of multiple developmental defects and embryonic lethality with loss of microsomal NADPHcytochrome P450 oxidoreductase. J Biol Chem 2002, 277:6536-6541.

2. Laue K, Janicke M, Plaster N, Sonntag C, Hammerschmidt M: Restriction of retinoic acid activity by Cyp26b1 is required for proper timing and patterning of osteogenesis during zebrafish development. Development 2008, 135:3775-3787.

3. White RJ, Nie Q, Lander AD, Schilling TF: Complex regulation of cyp26a1 creates a robust retinoic acid gradient in the zebrafish embryo. PLoS Biol 2007, 5:e304.

4. Uehara M, Yashiro K, Mamiya S, Nishino J, Chambon P, Dolle P, Sakai Y: CYP26A1 and CYP26C1 cooperatively regulate anterior-posterior patterning of the developing brain and the production of migratory cranial neural crest cells in the mouse. Dev Biol 2007, 302:399-411.

5. Hu MC, Hsu HJ, Guo IC, Chung BC: Function of Cyp11a1 in animal models. Mol Cell Endocrinol 2004, 215:95-100.

6. Otto DM, Henderson CJ, Carrie D, Davey M, Gundersen TE, Blomhoff R, Adams $\mathrm{RH}$, Tickle $\mathrm{C}$, Wolf $\mathrm{CR}$ : Identification of novel roles of the cytochrome p450 system in early embryogenesis: effects on vasculogenesis and retinoic Acid homeostasis. Mol Cell Biol 2003, 23:6103-6116.

7. Bair SR, Mellon SH: Deletion of the mouse P450c17 gene causes early embryonic lethality. Mol Cell Biol 2004, 24:5383-5390.

8. Stoilov I, Jansson I, Sarfarazi M, Schenkman JB: Roles of cytochrome p450 in development. Drug Metabol Drug Interact 2001, 18:33-55.

9. Alsop D, Vijayan MM: Molecular programming of the corticosteroid stress axis during zebrafish development. Comp Biochem Physiol A Mol Integr Physiol 2009, 153:49-54

10. Choudhary D, Jansson I, Stoilov I, Sarfarazi M, Schenkman JB: Expression patterns of mouse and human CYP orthologs (families 1-4) during development and in different adult tissues. Arch Biochem Biophys 2005, 436:50-61.

11. Hines RN: Ontogeny of human hepatic cytochromes P450. J Biochem Mol Toxicol 2007, 21:169-175.

12. Nebert DW, Dalton TP: The role of cytochrome P450 enzymes in endogenous signalling pathways and environmental carcinogenesis. Nat Rev Cancer 2006, 6:947-960.
13. Hernandez RE, Putzke AP, Myers JP, Margaretha L, Moens CB: Cyp26 enzymes generate the retinoic acid response pattern necessary for hindbrain development. Development 2007, 134:177-187.

14. Stoilov I: Cytochrome P450s: coupling development and environment. Trends Genet 2001, 17:629-632.

15. Waxman DJ: P450 gene induction by structurally diverse xenochemicals: central role of nuclear receptors CAR, PXR, and PPAR. Arch Biochem Biophys 1999, 369:11-23.

16. Handschin C, Meyer UA: Induction of drug metabolism: the role of nuclear receptors. Pharmacol Rev 2003, 55:649-673.

17. Fishman MC: Genomics. Zebrafish-the canonical vertebrate. Science 2001, 294:1290-1291.

18. Thisse B, Heyer V, Lux A, Alunni V, Degrave A, Seiliez I, Kirchner J, Parkhill JP Thisse C: Spatial and temporal expression of the zebrafish genome by large-scale in situ hybridization screening. Methods Cell Biol 2004, 77:505-519.

19. Ensembl Zebrafish. [http://www.ensembl.org/Danio_rerio/]

20. Choudhary D, Jansson I, Schenkman JB, Sarfarazi M, Stoilov I: Comparative expression profiling of 40 mouse cytochrome $\mathrm{P} 450$ genes in embryonic and adult tissues. Arch Biochem Biophys 2003, 414:91-100.

21. Nelson DR, Zeldin DC, Hoffman SM, Maltais LJ, Wain HM, Nebert DW: Comparison of cytochrome P450 (CYP) genes from the mouse and human genomes, including nomenclature recommendations for genes, pseudogenes and alternative-splice variants. Pharmacogenetics 2004, 14:1-18.

22. Guengerich FP: Human cytochrome P450 enzymes. In Cytochrome P450: Structure, Mechanism, and Biochemistry.. 3 edition. Edited by: Ortiz de Montellano PR. New York: Kluwer Academic; 2005:377-463.

23. Nelson DR, Koymans L, Kamataki T, Stegeman JJ, Feyereisen R, Waxman DJ, Waterman MR, Gotoh O, Coon MJ, Estabrook RW, et al: P450 superfamily: update on new sequences, gene mapping, accession numbers and nomenclature. Pharmacogenetics 1996, 6:1-42

24. Postlethwait $\mathrm{JH}$ : The zebrafish genome in context: ohnologs gone missing. J Exp Zool B Mol Dev Evol 2007, 308:563-577.

25. Wu KK, Liou JY: Cellular and molecular biology of prostacyclin synthase. Biochem Biophys Res Commun 2005, 338:45-52

26. Li YC, Chiang CW, Yeh HC, Hsu PY, Whitby FG, Wang LH, Chan NL: Structures of prostacyclin synthase and its complexes with substrate analog and inhibitor reveal a ligand-specific heme conformation change. J Biol Chem 2008, 283:2917-2926.

27. Hsu HJ, Liang MR, Chen CT, Chung BC: Pregnenolone stabilizes microtubules and promotes zebrafish embryonic cell movement. Nature 2006, 439:480-483

28. Hsu HJ, Hsiao P, Kuo MW, Chung BC: Expression of zebrafish cyp11a1 as a maternal transcript and in yolk syncytial layer. Gene Expr Patterns 2002, 2:219-222

29. Wang Y, Ge W: Cloning of zebrafish ovarian P450c17 (CYP17, 17alphahydroxylase/17, 20-lyase) and characterization of its expression in gonadal and extra-gonadal tissues. Gen Comp Endocrinol 2004 135:241-249.

30. Liu C, Yu L, Deng J, Lam PK, Wu RS, Zhou B: Waterborne exposure to fluorotelomer alcohol 6:2 $\mathrm{FTOH}$ alters plasma sex hormone and gene transcription in the hypothalamic-pituitary-gonadal (HPG) axis of zebrafish. Aquat Toxicol 2009, 93:131-137

31. Hoffmann $J$, Oris JT: Altered gene expression: a mechanism fo reproductive toxicity in zebrafish exposed to benzo[a]pyrene. Aquat Toxicol 2006, 78:332-340.

32. Zhou LY, Wang DS, Kobayashi T, Yano A, Paul-Prasanth B, Suzuki A, Sakai F, Nagahama $Y$ : A novel type of P450c17 lacking the lyase activity is responsible for C21-steroid biosynthesis in the fish ovary and head kidney. Endocrinology 2007, 148:4282-4291.

33. Zhou LY, Wang DS, Shibata Y, Paul-Prasanth B, Suzuki A, Nagahama Y: Characterization, expression and transcriptional regulation of P450c17-I and $-I I$ in the medaka, Oryzias latipes. Biochem Biophys Res Commun 2007, 362:619-625.

34. Kishida M, Callard GV: Distinct cytochrome P450 aromatase isoforms in zebrafish (Danio rerio) brain and ovary are differentially programmed and estrogen regulated during early development. Endocrinology 2001, 142:740-750.

35. Goto-Kazeto R, Kight KE, Zohar Y, Place AR, Trant JM: Localization and expression of aromatase mRNA in adult zebrafish. Gen Comp Endocrinol 2004, 139:72-84 
36. Callard GV, Tchoudakova AV, Kishida M, Wood E: Differential tissue distribution, developmental programming, estrogen regulation and promoter characteristics of cyp19 genes in teleost fish. J Steroid Biochem Mol Biol 2001, 79:305-314.

37. Stark K, Guengerich FP: Characterization of orphan human cytochromes P450. Drug Metab Rev 2007, 39:627-637.

38. Stark K, Wu ZL, Bartleson CJ, Guengerich FP: mRNA distribution and heterologous expression of orphan cytochrome P450 20A1. Drug Metab Dispos 2008, 36:1930-1937.

39. Craig TA, Sommer S, Sussman CR, Grande JP, Kumar R: Expression and regulation of the vitamin $\mathrm{D}$ receptor in the zebrafish, Danio rerio. J Bone Miner Res 2008, 23:1486-1496.

40. Reschly EJ, Bainy AC, Mattos JJ, Hagey LR, Bahary N, Mada SR, Ou J, Venkataramanan $\mathrm{R}$, Krasowski MD: Functional evolution of the vitamin D and pregnane $\times$ receptors. BMC Evol Biol 2007, 7:222.

41. White JA, Guo YD, Baetz K, Beckett-Jones B, Bonasoro J, Hsu KE, Dilworth FJ, Jones $\mathrm{G}$, Petkovich $\mathrm{M}$ : Identification of the retinoic acid-inducible alltrans-retinoic acid 4-hydroxylase. J Biol Chem 1996, 271:29922-29927.

42. Abu-Abed SS, Beckett BR, Chiba H, Chithalen JV, Jones G, Metzger D, Chambon P. Petkovich M: Mouse P450RAI (CYP26) expression and retinoic acid-inducible retinoic acid metabolism in F9 cells are regulated by retinoic acid receptor gamma and retinoid $\times$ receptor alpha. J Biol Chem 1998, 273:2409-2415.

43. White JA, Beckett-Jones B, Guo YD, Dilworth FJ, Bonasoro J, Jones G, Petkovich M: cDNA cloning of human retinoic acid-metabolizing enzyme (hP450RAl) identifies a novel family of cytochromes P450. J Biol Chem 1997, 272:18538-18541.

44. Ray WJ, Bain G, Yao M, Gottlieb DI: CYP26, a novel mammalian cytochrome $\mathrm{P} 450$, is induced by retinoic acid and defines a new family. J Biol Chem 1997, 272:18702-18708.

45. Gu X, Xu F, Song W, Wang X, Hu P, Yang Y, Gao X, Zhao Q: A novel cytochrome P450, zebrafish Cyp26D1, is involved in metabolism of alltrans retinoic acid. Mol Endocrinol 2006, 20:1661-1672.

46. Nelson DR: A second CYP26 P450 in humans and zebrafish: CYP26B1. Arch Biochem Biophys 1999, 371:345-347.

47. Spoorendonk KM, Peterson-Maduro J, Renn J, Trowe T, Kranenbarg S, Winkler C, Schulte-Merker S: Retinoic acid and Cyp26b1 are critical regulators of osteogenesis in the axial skeleton. Development 2008, 135:3765-3774

48. Kinkel MD, Sefton EM, Kikuchi Y, Mizoguchi T, Ward AB, Prince VE: Cyp26 enzymes function in endoderm to regulate pancreatic field size. Proc Natl Acad Sci USA 2009, 106:7864-7869.

49. Mast N, White MA, Bjorkhem I, Johnson EF, Stout CD, Pikuleva IA: Crystal structures of substrate-bound and substrate-free cytochrome P450 46A1, the principal cholesterol hydroxylase in the brain. Proc Natl Acad Sci USA 2008, 105:9546-9551.

50. Mast N, Norcross R, Andersson U, Shou M, Nakayama K, Bjorkhem I, Pikuleva IA: Broad substrate specificity of human cytochrome P450 46A1 which initiates cholesterol degradation in the brain. Biochemistry 2003 42:14284-14292

51. Russell DW, Halford RW, Ramirez DM, Shah R, Kotti T: Cholesterol 24hydroxylase: an enzyme of cholesterol turnover in the brain. Annu Rev Biochem 2009, 78:1017-1040.

52. Lamb DC, Kelly DE, Kelly SL: Molecular diversity of sterol 14alphademethylase substrates in plants, fungi and humans. FEBS Lett 1998, 425:263-265.

53. Rozman D, Stromstedt M, Tsui LC, Scherer SW, Waterman MR: Structure and mapping of the human lanosterol 14alpha-demethylase gene (CYP51) encoding the cytochrome P450 involved in cholesterol biosynthesis; comparison of exon/intron organization with other mammalian and fungal CYP genes. Genomics 1996, 38:371-381.

54. Morrison AM: Cloning, characterization, azole disruption and evolution of cytochrome P450 family 51 (CYP51) in aquatic animals. Harvard University, School of Public Health; 2003.

55. Weber GJ, Choe SE, Dooley KA, Paffett-Lugassy NN, Zhou Y, Zon LI: Mutantspecific gene programs in the zebrafish. Blood 2005, 106:521-530.

56. Yamazaki K, Teraoka H, Dong W, Stegeman JJ, Hiraga T: CDNA cloning and expressions of cytochrome P450 1A in zebrafish embryos. J Vet Med Sci 2002, 64:829-833.

57. Godard CA, Goldstone JV, Said MR, Dickerson RL, Woodin BR, Stegeman JJ: The new vertebrate CYP1C family: cloning of new subfamily members and phylogenetic analysis. Biochem Biophys Res Commun 2005 331:1016-1024.

58. Jönsson ME, Jenny MJ, Woodin BR, Hahn ME, Stegeman JJ: Role of AHR2 in the expression of novel cytochrome P450 1 family genes, cell cycle genes, and morphological defects in developing zebra fish exposed to 3,3',4,4',5-pentachlorobiphenyl or 2,3,7,8-tetrachlorodibenzo-p-dioxin. Toxicol Sci 2007, 100:180-193.

59. Goldstone JV, Jönsson ME, Behrendt L, Woodin BR, Jenny MJ, Nelson DR, Stegeman JJ: Cytochrome P450 1D1: a novel CYP1A-related gene that is not transcriptionally activated by PCB126 or TCDD. Arch Biochem Biophys 2009, 482:7-16.

60. Goldstone JV, Goldstone HM, Morrison AM, Tarrant A, Kern SE, Woodin BR, Stegeman JJ: Cytochrome P450 1 genes in early deuterostomes (tunicates and sea urchins) and vertebrates (chicken and frog): origin and diversification of the CYP1 gene family. Mol Biol Evol 2007, 24:2619-2631.

61. Kawai YK, Ikenaka Y, Fujita S, Ishizuka M: The CYP1 D subfamily of genes in mammals and other vertebrates. Mamm Genome 2010, 21:320-329.

62. Jönsson ME, Orrego R, Woodin BR, Goldstone JV, Stegeman JJ: Basal and 3, $3^{\prime}, 4,4^{\prime}, 5$-pentachlorobiphenyl-induced expression of cytochrome P450 1A, 1B and 1C genes in zebrafish. Toxicol Appl Pharmacol 2007, 221:29-41.

63. Scornaienchi ML, Thornton C, Willett KL, Wilson JY: Cytochrome P450 mediated 17 \{beta\}-estradiol metabolism in zebrafish (Danio rerio). $J$ Endocrinol 2010

64. Scornaienchi ML, Thornton C, Willett KL, Wilson JY: Functional differences in the cytochrome P450 1 family enzymes from Zebrafish (Danio rerio) using heterologously expressed proteins. Arch Biochem Biophys 2010, 502:17-22.

65. Cheng JB, Motola DL, Mangelsdorf DJ, Russell DW: De-orphanization of cytochrome P450 2R1: a microsomal vitamin D 25-hydroxilase. J Biol Chem 2003, 278:38084-38093.

66. Strushkevich N, Usanov SA, Plotnikov AN, Jones G, Park HW: Structural analysis of CYP2R1 in complex with vitamin D3. J Mol Biol 2008, 380:95-106.

67. Karlgren M, Backlund M, Johansson I, Oscarson M, Ingelman-Sundberg M: Characterization and tissue distribution of a novel human cytochrome P450-CYP2U1. Biochem Biophys Res Commun 2004, 315:679-685.

68. Chuang SS, Helvig C, Taimi M, Ramshaw HA, Collop AH, Amad M, White JA, Petkovich M, Jones G, Korczak B: CYP2U1, a novel human thymus- and brain-specific cytochrome P450, catalyzes omega- and (omega-1)hydroxylation of fatty acids. J Biol Chem 2004, 279:6305-6314.

69. Goldstone JV, Hamdoun A, Cole BJ, Howard-Ashby M, Nebert DW, Scally M, Dean M, Epel D, Hahn ME, Stegeman JJ: The chemical defensome: environmental sensing and response genes in the Strongylocentrotus purpuratus genome. Dev Biol 2006, 300:366-384

70. Oleksiak MF, Wu S, Parker C, Karchner SI, Stegeman JJ, Zeldin DC: Identification, functional characterization, and regulation of a new cytochrome P450 subfamily, the CYP2Ns. J Biol Chem 2000, 275:2312-2321.

71. Oleksiak MF, Wu S, Parker C, Qu W, Cox R, Zeldin DC, Stegeman JJ: Identification and regulation of a new vertebrate cytochrome P450 subfamily, the CYP2Ps, and functional characterization of CYP2P3, a conserved arachidonic acid epoxygenase/19-hydroxylase. Arch Biochem Biophys 2003, 411:223-234.

72. Edler D, Stenstedt $K$, Ohrling $K$, Hallstrom M, Karlgren M, IngelmanSundberg M, Ragnhammar P: The expression of the novel CYP2W1 enzyme is an independent prognostic factor in colorectal cancer - a pilot study. Eur J Cancer 2009, 45:705-712.

73. Karlgren M, Gomez A, Stark K, Svard J, Rodriguez-Antona C, Oliw E, Bernal ML, Ramon y Cajal S, Johansson I, Ingelman-Sundberg M: Tumorspecific expression of the novel cytochrome P450 enzyme, CYP2W1. Biochem Biophys Res Commun 2006, 341:451-458.

74. Yoshioka H, Kasai N, Ikushiro S, Shinkyo R, Kamakura M, Ohta M, Inouye K, Sakaki T: Enzymatic properties of human CYP2W1 expressed in Escherichia coli. Biochem Biophys Res Commun 2006, 345:169-174.

75. Wang-Buhler JL, Lee SJ, Chung WG, Stevens JF, Tseng HP, Hseu TH, Hu CH, Westerfield M, Yang YH, Miranda CL, Buhler DR: CYP2K6 from zebrafish (Danio rerio): cloning, mapping, developmental/tissue expression, and aflatoxin B1 activation by baculovirus expressed enzyme. Comp Biochem Physiol C Toxicol Pharmacol 2005, 140:207-219. 
76. Schlenk D, Furnes B, Zhou X, Debusk BC: Cloning and sequencing of cytochrome P450 2X1 from channel catfish (Ictalurus punctatus). Mar Environ Res 2002, 54:391-394.

77. Mosadeghi S, Furnes B, Matsuo AY, Schlenk D: Expression and characterization of cytochrome P450 2X1 in channel catfish (Ictalurus punctatus). Biochim Biophys Acta 2007, 1770:1045-1052.

78. Birney E, Clamp M, Durbin R: GeneWise and Genomewise. Genome Res 2004, 14:988-995.

79. Corley-Smith GE, Su HT, Wang-Buhler JL, Tseng HP, Hu CH, Hoang T, Chung WG, Buhler DR: CYP3C1, the first member of a new cytochrome P450 subfamily found in zebrafish (Danio rerio). Biochem Biophys Res Commun 2006, 340:1039-1046.

80. Tseng HP, Hseu TH, Buhler DR, Wang WD, Hu CH: Constitutive and xenobiotics-induced expression of a novel CYP3A gene from zebrafish larva. Toxicol Appl Pharmacol 2005, 205:247-258.

81. Qiu H, Taudien S, Herlyn H, Schmitz J, Zhou Y, Chen G, Roberto R, Rocchi M, Platzer M, Wojnowski L: CYP3 phylogenomics: evidence for positive selection of CYP3A4 and CYP3A7. Pharmacogenet Genomics 2008, 18:53-66.

82. Bresolin T, de Freitas Rebelo M, Celso Dias Bainy A: Expression of PXR, CYP3A and MDR1 genes in liver of zebrafish. Comp Biochem Physio/ C Toxicol Pharmacol 2005, 140:403-407.

83. James MO, Lou Z, Rowland-Faux L, Celander MC: Properties and regional expression of a CYP3A-like protein in channel catfish intestine. Aquat Toxicol 2005, 72:361-371.

84. Hardwick JP: Cytochrome P450 omega hydroxylase (CYP4) function in fatty acid metabolism and metabolic diseases. Biochem Pharmacol 2008, 75:2263-2275

85. Kalsotra A, Strobel HW: Cytochrome P450 4F subfamily: at the crossroads of eicosanoid and drug metabolism. Pharmacol Ther 2006, 112:589-611.

86. Nakano M, Kelly EJ, Rettie AE: Expression and characterization of CYP4V2 as a fatty acid omega-hydroxylase. Drug Metab Dispos 2009, 37:2119-2122.

87. Sabourault C, Berge J, Lafaurie M, Girard JP, Amichot M: Molecular cloning of a phthalate-inducible CYP4 gene (CYP4T2) in kidney from the sea bass, Dicentrarchus labrax. Biochem Biophys Res Commun 1998, 251:213-219.

88. Liu Y, Wang J, Liu Y, Zhang $H, X u M$, Dai J: Expression of a novel cytochrome P450 4T gene in rare minnow (Gobiocypris rarus) following perfluorooctanoic acid exposure. Comp Biochem Physiol C Toxicol Pharmacol 2009, 150:57-64.

89. Aryee MJ, Gutierrez-Pabello JA, Kramnik I, Maiti T, Quackenbush J: An improved empirical bayes approach to estimating differential gene expression in microarray time-course data: BETR (Bayesian Estimation of Temporal Regulation). BMC Bioinformatics 2009, 10:409.

90. Wang L, Yao J, Chen L, Chen J, Xue J, Jia W: Expression and possible functional roles of cytochromes P450 2J1 (zfCyp 2J1) in zebrafish. Biochem Biophys Res Commun 2007, 352:850-855.

91. Ben-Dor A, Shamir R, Yakhini Z: Clustering gene expression patterns. Comput Biol 1999, 6:281-297.

92. Sato $Y$, Hashiguchi $Y$, Nishida M: Temporal pattern of loss/persistence of duplicate genes involved in signal transduction and metabolic pathways after teleost-specific genome duplication. BMC Evol Biol 2009, 9:127.

93. Kishida M, McLellan M, Miranda JA, Callard GV: Estrogen and xenoestrogens upregulate the brain aromatase isoform (P450aromB) and perturb markers of early development in zebrafish (Danio rerio) Comp Biochem Physiol B Biochem Mol Biol 2001, 129:261-268.

94. Tchoudakova A, Kishida M, Wood E, Callard GV: Promoter characteristics of two cyp19 genes differentially expressed in the brain and ovary of teleost fish. J Steroid Biochem Mol Biol 2001, 78:427-439.

95. Cheshenko K, Brion F, Le Page Y, Hinfray N, Pakdel F, Kah O, Segner H, Eggen Rl: Expression of zebra fish aromatase cyp19a and cyp19b genes in response to the ligands of estrogen receptor and aryl hydrocarbon receptor. Toxicol Sci 2007, 96:255-267.

96. Nelson DR: Comparison of P450 s from human and fugu: 420 million years of vertebrate P450 evolution. Arch Biochem Biophys 2003, 409:18-24.

97. McArthur AG, Hegelund T, Cox RL, Stegeman JJ, Liljenberg M, Olsson U, Sundberg P, Celander MC: Phylogenetic analysis of the cytochrome P450 3 (CYP3) gene family. J Mol Evol 2003, 57:200-211.

98. Celander M, Buhler DR, Forlin L, Goksoyr A, Miranda CL, Woodin BR, Stegeman JJ: Immunochemical relationships of cytochrome P4503A-like proteins in teleost fish. Fish Physiology and Biochemistry 1996, 15:323-332.
99. Stegeman JJ: Polynuclear aromatic hydrocarbons and their metabolism in the marine environment. In Polycyclic Hydrocarbons and Cancer. Volume 3. Edited by: Gelboin HV, Ts'o POP. New York: Academic Press; 1981:1-60.

100. Schlezinger JJ, Keller J, Verbrugge LA, Stegeman JJ: 3,3',4,4'Tetrachlorobiphenyl oxidation in fish, bird and reptile species: relationship to cytochrome $\mathrm{P} 4501 \mathrm{~A}$ inactivation and reactive oxygen production. Comp Biochem Physiol C Toxicol Pharmacol 2000, 125:273-286.

101. Prasad JC, Goldstone JV, Camacho CJ, Vajda S, Stegeman JJ: Ensemble modeling of substrate binding to cytochromes P450: analysis of catalytic differences between CYP1A orthologs. Biochemistry 2007, 46:2640-2654.

102. Trant JM, Gavasso S, Ackers J, Chung BC, Place AR: Developmental expression of cytochrome P450 aromatase genes (CYP19a and CYP19b) in zebrafish fry (Danio rerio). J Exp Zool 2001, 290:475-483.

103. Reijntjes S, Rodaway A, Maden M: The retinoic acid metabolising gene, CYP26B1, patterns the cartilaginous cranial neural crest in zebrafish. Int $J$ Dev Biol 2007, 51:351-360.

104. Sirbu IO, Gresh L, Barra J, Duester G: Shifting boundaries of retinoic acid activity control hindbrain segmental gene expression. Development 2005, 132:2611-2622.

105. Sawyer SJ, Gerstner KA, Callard GV: Real-time PCR analysis of cytochrome P450 aromatase expression in zebrafish: gene specific tissue distribution, sex differences, developmental programming, and estrogen regulation. Gen Comp Endocrinol 2006, 147:108-117.

106. Ito $Y$, Matsuda Y, Suzuki T: Effects of 3,4-dichloroaniline on expression of ahr2 and cyp1a1 in zebrafish adults and embryos. Comp Biochem Physiol C Toxicol Pharmacol 152:189-194.

107. Goldstone HM, Stegeman JJ: A revised evolutionary history of the CYP1A subfamily: gene duplication, gene conversion, and positive selection. J Mol Evol 2006, 62:708-717.

108. Eddy SR: Profile hidden Markov models. Bioinformatics 1998, 14:755-763.

109. Edgar RC: MUSCLE: a multiple sequence alignment method with reduced time and space complexity. BMC Bioinformatics 2004, 5:113.

110. Stamatakis A: RAxML-VI-HPC: maximum likelihood-based phylogenetic analyses with thousands of taxa and mixed models. Bioinformatics 2006, 22:2688-2690.

111. Whelan S, Goldman N: A general empirical model of protein evolution derived from multiple protein families using a maximum-likelihood approach. Mol Biol Evol 2001, 18:691-699.

112. Westerfield M: The zebrafish book. A guide for the laboratory use of zebrafish (Danio rerio). 4 edition. Eugene, OR: Univ. of Oregon Press; 2000.

113. Schadt EE, Li C, Ellis B, Wong WH: Feature extraction and normalization algorithms for high-density oligonucleotide gene expression array data J Cell Biochem Suppl 2001, , Suppl 37: 120-125.

114. Schadt EE, Li C, Su C, Wong WH: Analyzing high-density oligonucleotide gene expression array data. J Cell Biochem 2000, 80:192-202.

115. Li C, Wong WH: Model-based analysis of oligonucleotide arrays: expression index computation and outlier detection. Proc Natl Acad Sci USA 2001, 98:31-36.

116. Saeed Al, Sharov V, White J, Li J, Liang W, Bhagabati N, Braisted J, Klapa M, Currier T, Thiagarajan M, et al: TM4: a free, open-source system for microarray data management and analysis. Biotechniques 2003, 34:374-378.

117. Lai WW, Hsiao PH, Guiguen Y, Chung BC: Cloning of zebrafish cDNA for 3beta-hydroxysteroid dehydrogenase and P450scc. Endocr Res 1998, 24:927-931.

118. Zhao Q, Dobbs-McAuliffe B, Linney E: Expression of cyp26b1 during zebrafish early development. Gene Expr Patterns 2005, 5:363-369.

119. Gu X, Xu F, Wang X, Gao X, Zhao Q: Molecular cloning and expression of a novel CYP26 gene (cyp26d1) during zebrafish early development. Gene Expr Patterns 2005, 5:733-739.

doi:10.1186/1471-2164-11-643

Cite this article as: Goldstone et al:: Identification and developmental expression of the full complement of Cytochrome P450 genes in Zebrafish. BMC Genomics 2010 11:643. 